\title{
Analysis and Classification of Shape-Changing Interfaces for Design and Application-based Research
}

\author{
MIRIAM STURDEE and JASON ALEXANDER, Lancaster University, United Kingdom
}

Shape-changing interfaces are physically tangible, interactive devices, surfaces or spaces which allow for rich, organic and novel experiences with computational devices. Over the last fifteen years, research has produced functional prototypes over many use-applications, and reviews have identified themes and possible future directions-but have not yet looked at possible design or application based research. Here we gather this information together to provide a reference for designers and researchers wishing to build upon existing prototyping work, using synthesis and discussion of existing shape-changing interface reviews and comprehensive analysis and classification of 84 shape-changing interfaces. Eight categories of prototype are identified, alongside recommendations for the field.

CCS Concepts: • Human-centered computing $\rightarrow$ Human computer interaction (HCI); Interaction devices; Interface design prototyping;

Additional Key Words and Phrases: Shape-changing interfaces, TUI, GTUI, application design, classification

ACM Reference Format:

Miriam Sturdee and Jason Alexander. 2018. Analysis and Classification of Shape-Changing Interfaces for Design and Application-based Research. ACM Comput. Surv. 51, 1, Article 2 (January 2018), 31 pages. https: //doi.org/10.1145/3143559

\section{INTRODUCTION}

Shape-changing interfaces are physically geometric dynamic computational systems which also support an additional range of inputs (such as touch and shape-deformation) and outputs (such as light or sound). Prototypes of this nature are becoming more common within HCI, as advances are made in Shape Changing Materials/Alloys (SCM/SMAs), flexible displays and actuation techniques, thus supporting increasingly more detailed and interactive user experiences. It is feasible to imagine that within the next 50 years, such devices will augment or replace the pervasive $2 \mathrm{D}$ screens with which we currently navigate digital space.

Now that the field is maturing quickly, with highly interactive, dynamic and usable prototypes in abundance, we must think beyond the initial test-phase and toward designing meaningful applications (alongside the already identified interactions) for tangible future input and output. Although several research teams have begun to explore and discuss this exciting future, e.g. Roudaut [80] and Jansen [38], at present many applications are either pre-existing program types (such as music players or book readers) [52] or designed for one specific iteration of a device as a demonstration of its capabilities [53]. However, it is because of these explicit investigations, that we have a solid starting point for the evolution of these interfaces. The difficulty lies in creating content for such diverse and multi-dimensional devices.

Poupyrev [76] suggested in 2007 that future research might systematically investigate applications of actuated devices for various uses, outlining how our notion of pixels might further develop as dimensionality is added to graphical information (see Figure 1). Additionally, whilst researchers

Authors' address: Miriam Sturdee; Jason Alexander, Lancaster University, Infolab21, South Drive, Lancaster University, Bailrigg, Lancaster, LA1 4WA, United Kingdom, m.sturdee@lancaster.ac.uk,j.alexander@lancaster.ac.uk.

() 2018 Association for Computing Machinery.

This is the author's version of the work. It is posted here for your personal use. Not for redistribution. The definitive Version of Record was published in ACM Computing Surveys, https://doi.org/10.1145/3143559. 
have started to try and make sense of the design space of shape-changing interfaces, where multiple dimensions must be considered at the same time [11,50,102] thus far it appears that there has been little consideration for designing generic applications for shape changing devices, as we might do for standard 2D UIs. Speculative work relating to solving current hardware problems, or the qualities of future materials [35], leaves a space in between the prototypical present, and the near future of marketable shape changing products.

The basis for this work is the significant body of research on gestures and interactions with shape-changing displays [23, 100] (for example), but the results of these studies have not yet been channelled into a consolidated, cross-paper set of guidelines for designers. There are even prototypes designed specifically for the act of prototyping itself $[28,32]$ to help designers make the first step, but there appears to be no united front on where that first step falls.

In order to assist researchers and designers in continuing to examine the current state of the field and the potential applications, this review collates some of the existing theoretical work on designing for shape-change-taken from several reviews [11, 50, 69, 79], interaction studies [32, 67], prototyping tools for shape-change [28] and general prototype papers-to create a comprehensive overview of dimensionality within shape-changing interfaces. The resulting amalgam from these detailed reviews (looking at such features as spatiality, temporality, interaction, and hardware) is then applied directly to existing work on these prototypes, so that categories of device are formed. These categories are discussed in relation to the design space, existing research, and limitations. The discussion looks at supporting application design, hybridisation, limitation in design, future use cases, emotionality and user experience, future use cases, perception theory, the notion of temporal design and ethics, whilst considering how speculation might inform future work.

Tangible User Interfaces (TUIs) are swiftly making inroads into retail reality (witness Nokia's Kinetic Device [44]), merging with shape changing displays to create proto-GTUIs (GraphicalTangible User Interfaces). Holman \& Vertegaal [33] comment on the complexity of designing for this new generation of shape changing interface/display, stating that all physics acting upon displays, including their shape, will be used to manipulate information. So we must look not only to the manipulation of physical form to design our applications, but also to the other senses and beyond. The following work is the first consolidated review of shape-changing interface theory, and also the first to provide a comprehensive analysis and categorisation of existing prototypes. The latter is necessary in order to begin to formalise design for the field and should be used to inform detailed application design for current shape-changing interfaces in the research context.

This paper contributes a contemporary meta-analysis of shape changing design theory, a detailed database of shape-changing prototypes, and a categorisation of types of shape-changing interface (Enhanced 2D, Bendable, Paper \& Cloth, Elastic \& Inflatable, Actuated, Liquid, Malleable and Hybrid). The aim of the paper is to assist researchers interested in contributing novel prototypes and their applications to the field, and designers who wish to gain knowledge of current hardware to begin to create meaningful deformable applications for real world iterations of these devices. The main goal of this review is to set the stage for application design for shape-changing interfaces by providing a reference guide for each interface type and their associated interactions, with which we can inspire real use cases for existing prototypes and look beyond this, to the commercial future of shape-changing interfaces.

\section{RELATED WORK}

There is a well-cited and succinct body of work that outlines the current design and mechanical aspirations of the shape-changing interface field. These are outlined in this section, and relate to the consolidated dimensions in Figure 1. The contribution of this paper in relation to previous work is in its thorough review of the available literature, combined analysis of leading papers in the field, 
novelty of the consolidation of attributes and subsequent categorisation of prototypes within this context. This is the first time the field of shape-change has been looked at in as much breadth and depth, and builds upon the valuable contributions made by the researchers discussed below.

Rasmussen's review of shape changing interfaces [79] suggests that there is a great deal of research into hardware, but that the design possibility of this space is an underexplored direction. If, as Vallgårda [102] states, a "new expectation of the computer is already being formed" we therefore need to rise to the challenge of meeting this expectation with tangible shape changing interfaces that will appeal to the next generation of users. Vallgårda creates a baseline for the new type of interaction design necessary for shape changing interfaces, where temporality meets the physical and the interactive possibilities of such devices. This 'trinity' should form the cornerstone for any designer wishing to make a start in this area.

Kwak [50], held boot-camps for industrial designers to create platforms for prototyping design for shape change, meaning that future designers can explore basic transitions and actions which then form the basis for the nascent application of shape changing interfaces and displays. Six prototyping tools were identified from an initial selection of ten which cover a range of deformations and actions (Piega, Gato, Yeti, Fantom, Squeezy \& Bulge). These prototyping devices mirror the most common deformation styles found in shape changing interfaces (bar those that make use of 2D flexible computers), and thus provide a neat overview of deformation styles, which can be aptly applied to the overview of shape-changing interfaces.

From a point of view based on the theory of Non-Uniform Rational B-Splines, Roudaut et al. [80] propose a framework for shape-resolution - aimed at assisting engineers in creating high resolution displays. This framework is only as good as the technology allows though, and its advanced features will need to be applied gradually. It also only applies to those mechanisms which can be thought of as having nodes/loci of control (as seen in a mesh overlay), and thus only applies in part to shape-changing materials, which also require thinking in other dimensions which may not be so constrained.

Coelho et al's review [11] focuses on all possible realities for shape change in a speculative manner, and further provides an interesting overview of the field as it was in 2011. By combining the multiple dimensionality of shape changing interfaces, they attempt to begin construction of a 'soft' mechanical alphabet for HCI (after 18th century engineer Polhem) with which designers can orientate themselves for this conceptually complex research area. This notion supports this review in regards to the need for a modular design theory for those wishing to engage in application design for GTUIs.

From the side of programming interactions, there has been a start on creating a specific languages for designing shape-changing interactions (based on existing Shader languages [107]), but any advances in programming will still need to be relatable to designers. At present, researchers must have a firm grounding in programming, electronics, and mechanical engineering to engage with shape changing interfaces, although this might change in the wake of the recent surge in interest toward interdisciplinary study.

\section{CONSOLIDATION OF SHAPE-CHANGE THEMES}

A meta-analysis of papers from Coelho [11], Roudaut [80], Taher [94], Rasmussen [79] and Kwak [50] was conducted, alongside complimentary information from Nørgaard [69], Schmid [85] and Hardy [28] in order to create a comprehensive overview of the state of shape-change as it stands at present. These papers were chosen as they covered the breadth of the area in terms of interfaces, although SCM papers were consulted alongside to ensure that all dimensions of change were covered. The categorisations provided by each researcher have been mapped alongside one another in Figure 1. Following analysis of these papers, it was also found that the types of 


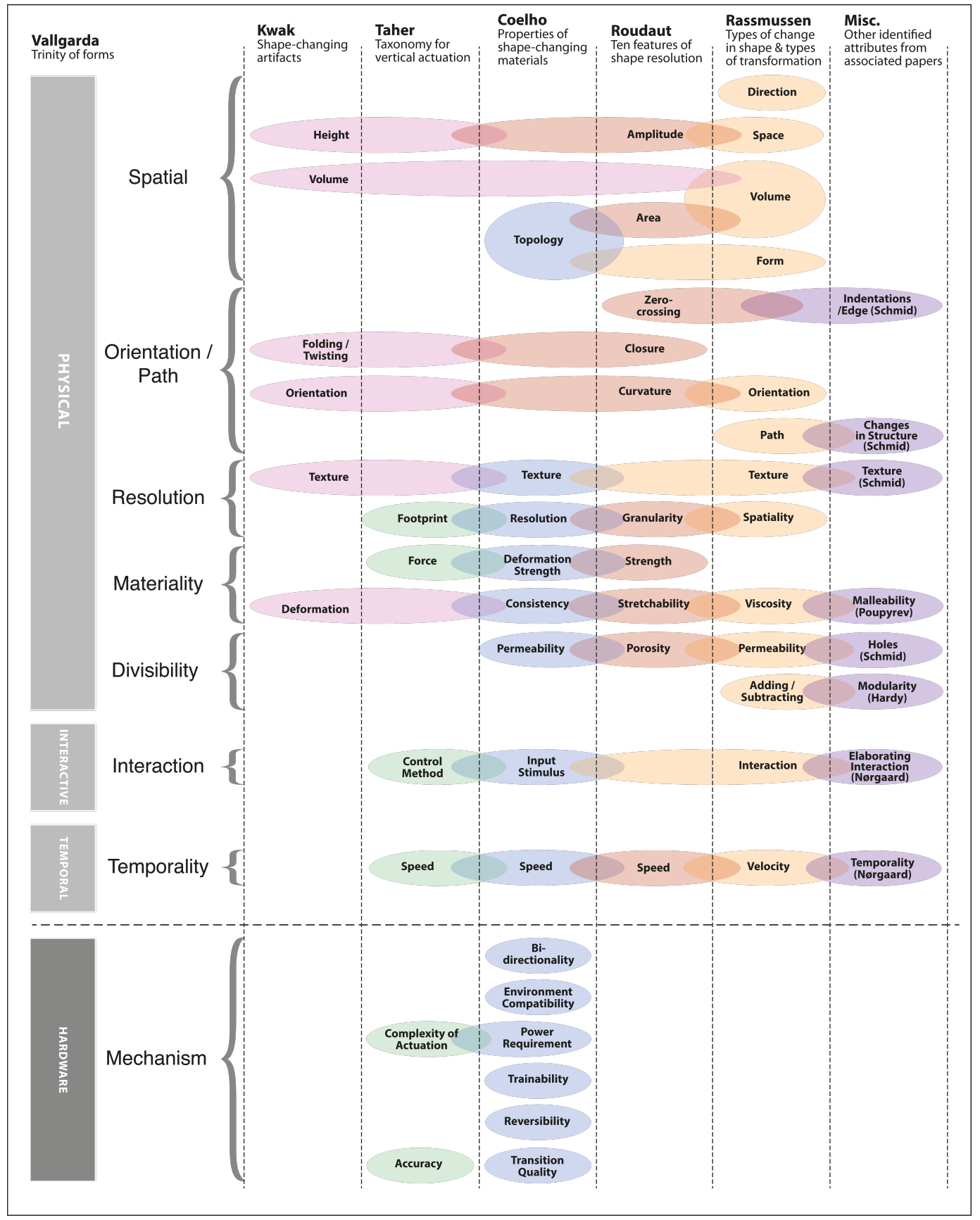

Fig. 1. Meta-analysis of shape-change review papers, taxonomies and categorisations 
change which one needs to consider when thinking around the topic of design followed closely to Vallgårda's [102] "Trinity of Forms". A separate area for back-end, hardware considerations was created, in order to relate back to the hardware and mechanism of shape-changing interfaces, rather than the pure theory.

To summarise the sections in Figure 1, the Spatial section relates to topology, expansion, height and spread of the interface display area; Orientation/Path toward folding and turning abilities of devices; Resolution toward textural and pixel quality (which may go hand-in-hand according to Nørgaard [69] - a high enough shape resolution means that the generation of texture is a given); Materiality concerns the pliability and strength of the surface; and Divisibility, the separation of component parts or ability of a material to let through matter.

The interactive qualities of a shape-changing interface are not expanded in this diagram, as interaction is a multi-faceted aspect of a GTUI and requires a more detailed overview (see [79]). Rasmussen et al. suggest three types of interaction in shape-change: direct, indirect and remote. These types have been used in applying classification to the existing prototypes in Tables 1-8, as well as including types of input/output. These are discussed in the next section.

Temporality is a relatively new concept in design, but known to those working on shapechange and therefore is vital to any theorist hoping to create content for these devices. Finally, the mechanistic aspects, or hardware in a device are held separate, but nonetheless accountable to the interface itself, for these component parts hold the key to the outer and inner limits of what is possible now, and in the future.

By examining the ways in which these dimensions map alongside each other and interact, we have ensured that we have an easily accessible summary from which we can begin to formalise the nature of this area - all these categories are discussed in more detail in section 4 . The information in Tables 1 to 8 is based on this summary, and the nature of existing devices in relation to the wider theory-based dimensions is discussed later in the paper.

\section{APPLICATION TO EXISTING PROTOTYPES}

Having condensed current theory into a meaningful summary (Figure 1), the next stage was to apply this method of analysis to existing prototypes in order to gain an overview of the current state of the art with regards to design and applications. The category descriptions in the previous section have been changed to reflect existing deformation types (rather than future possibilities), and the interactive aspect constructed during analyses of the literature. It also proved of further use to add fields to the following tables which give additional information (such as $2 \mathrm{D} / 2.5 \mathrm{D} / 3 \mathrm{D}$ ).

Tables 1-8 provide a comprehensive overview of 84 existing shape changing prototype interfaces from the past 16 years, as they were at time of writing. This builds upon Rasmussen's review of 44 papers on shape-changing interfaces [79], but with a more refined criteria for inclusion and an tabulated analysis which compares the field. Figure 3 provides a graphical overview of this categorisation in order to compare between groups at a glance. Further to this, a summary table (Table 9) outlines the main features of the display categories.

\subsection{Inclusion Criteria}

The inclusion criteria are that: each prototype must be interactive (have at least one human user), have at least one type of input and output occurring on the same surface; and that each included prototype must be composed of a malleable material or deformable mechanism. These criteria mean that ShapePhone [18] and Behind-the-Tablet famming [18] are exempt (because ShapePhone is an input only deformable phone prototype with no display mechanism, and Behind-the-Tablet famming separates deformation area and display) but that Tunable Clay is included as the image is projected directly onto the malleable surface [18]. The same reasoning applies to Tangible User 
Interface (TUI) input-only devices such as BendID [67] and AR-fig [4]. Additionally, although Asif Khan's Megafaces [43] is an exemplar of an hydraulic actuated display - reflects user input (digital photography and 3D image extrapolation) - it does not behave as a true interface (as described above) in its current iteration. The user in this case is passive, and unable to dictate or influence the output.

Another type of shape-changing prototype that is excluded is Guo et al's Garden Agua [27] despite being described as shape-changing display in the literature - as it deals only with moveable solid objects and not surface deformation. The same premise also applies to Ariel Tunes [3] due to the modular and limited nature of its current form-based output. Despite the pixel-like nature of the floating balls in Ariel Tunes, the display supports only one type of interaction and one type of output. This is not to say that future iterations of such mechanisms may not fulfil the criteria outlined here. Finally, where there is more than one iteration of the same prototype, the most recent is included, unless a significant change to the usage has been implemented - such as FuSA 2 [63]

The reasoning behind setting strict inclusion criteria is that tangible input devices require design only for existing 2D output, which is a well established field, hence the same surface must be utilised in order to establish something novel. The same also applies to non-deformable surfaces there is no need to establish a new framework of analysis or design. It is also worth noting that definitions of "interface" within shape-change differ between researchers, the criterion here are not intended to exclude without reason, merely to draw a line around what a shape-changing interface is for the purpose of analysis. Future work may expand on this analysis to look at the wider field of tangible TUIs and shape-displays within the overview provided here.

\subsection{Dimensions of Shape-Change}

In applying existing prototypes to category headers, we further condensed the dimensions from within Figure 1, and also identified types of prototype hardware currently used in the literature. The resulting fields of classification are discussed below in order to clarify their use.

\subsection{Hardware}

The mechanism, or hardware, of each device is directly linked to its shape-changing properties (see Figure 1). As advances are made in the field of shape-change, it is anticipated that the list of hardware types will grow. As of now, 24 basic hardware composites have been identified from current prototypes, which can be combined to create amalgams of shape and display. Each table outlines a primary and/or secondary mechanism where this is integral to the interaction of the prototype. Incidental structural materials, such as latex or wood, are omitted from this list.

Some of the dimensions of shape-changing interfaces were identified at the consolidation stage, but either do not apply to existing prototypes in a quantifiable manner (i.e. power requirement is something to be considered at the commercialisation phase) or would require additional levels of detail and discussion for each individual prototype which are not possible within the scope of this paper.

4.3.1 Bi-Directionality. Whereas Coelho stated that bi-directionality is specifically important for designers [11] it is not an exclusive construct within shape-changing prototypes, and thus has not been applied to the list. Bi-directionality refers to the properties of a material/device to physically change shape in the same way when deformed by a user, and when self-actuating. This is important during the design process as it has an effect on other material properties of the interaction surface, and the interactions a user will have with the interface (i.e. non-bi-directionality might be seen in the case of clay-based interfaces where the user can deform the surface, but the surface itself is passive, in which case it must be manually "re-set"). 

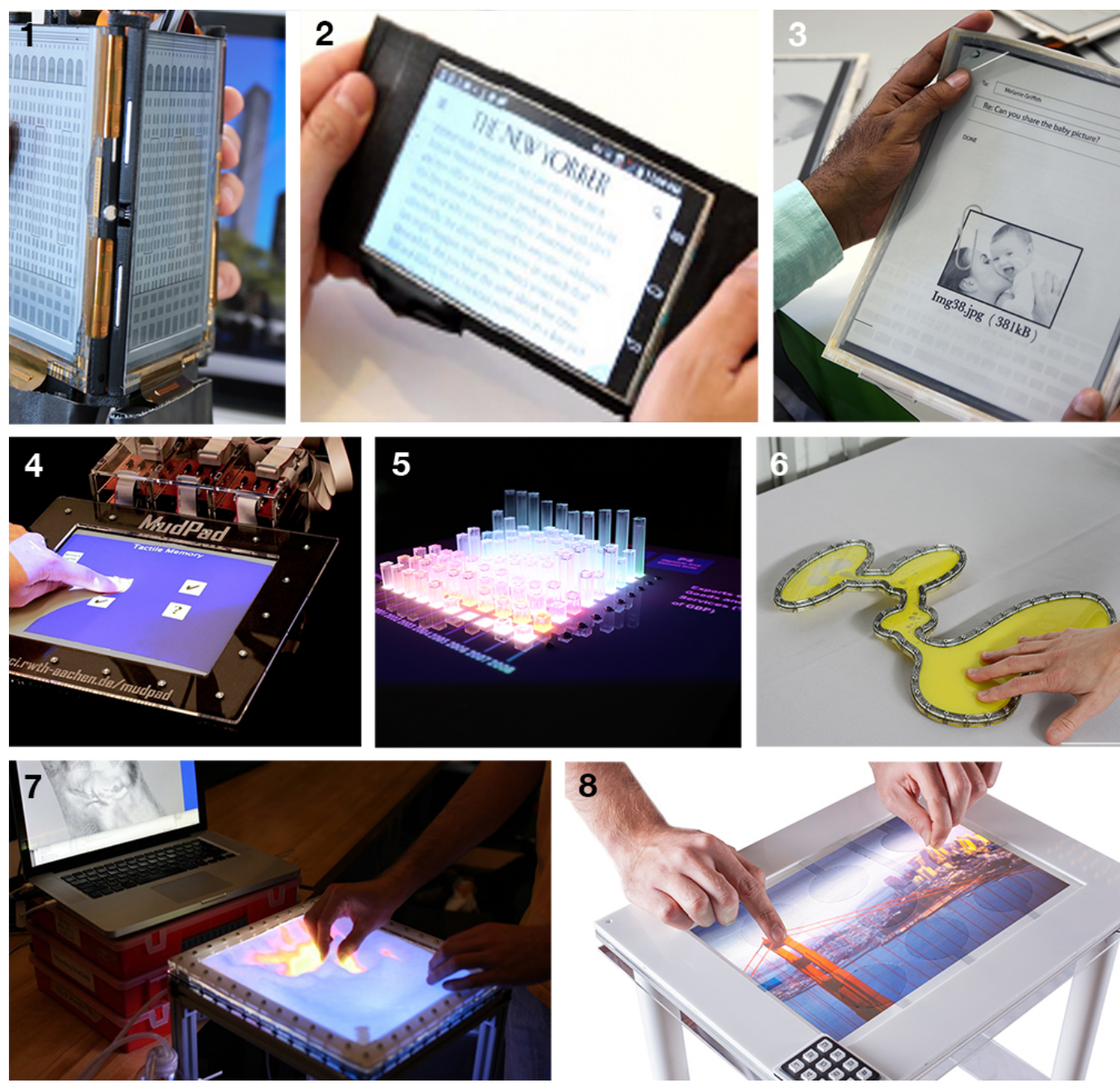

Fig. 2. Current prototypes corresponding to the 8 categories of shape-changing interface identified by this review: 1. Enhanced 2D - PaperFold [25]; 2. Bendable - Reflex [91]; 3. Papers \& Cloths - PaperTab [99]; 4. Elastics \& Inflatables - Mudpad [39]; 5. Actuated - Emerge [93]; 6. Liquids - Linetic [48]; 7. Malleables Tunable Clay [18] (courtesy of MIT Media Lab Tangible Media Group); 8. Hybrids - TableHop [83] (courtesy of Deepak Sahoo)

Most examples have varying inputs and outputs but they are not always linked, for example, form-input is not always directly related to form-output by the mechanism, such as with Paddle [77] which utilises purely user-controlled deformation. For Paddle to exhibit bi-directionality, it would have to be able to deform itself in response to some other form of input, such as a telephone call activating a form-state.

Some examples do exhibit bi-directionality in limited ways however: ShapeClip [28] is bidirectional in respect to the input/output of tangible form and light, but can only react to imagebased light input, not produce it (this limitation is addressed by ShapeCanvas [15] however, which uses the same base mechanism). The same applies to LightCloth [30] which accepts/projects light 


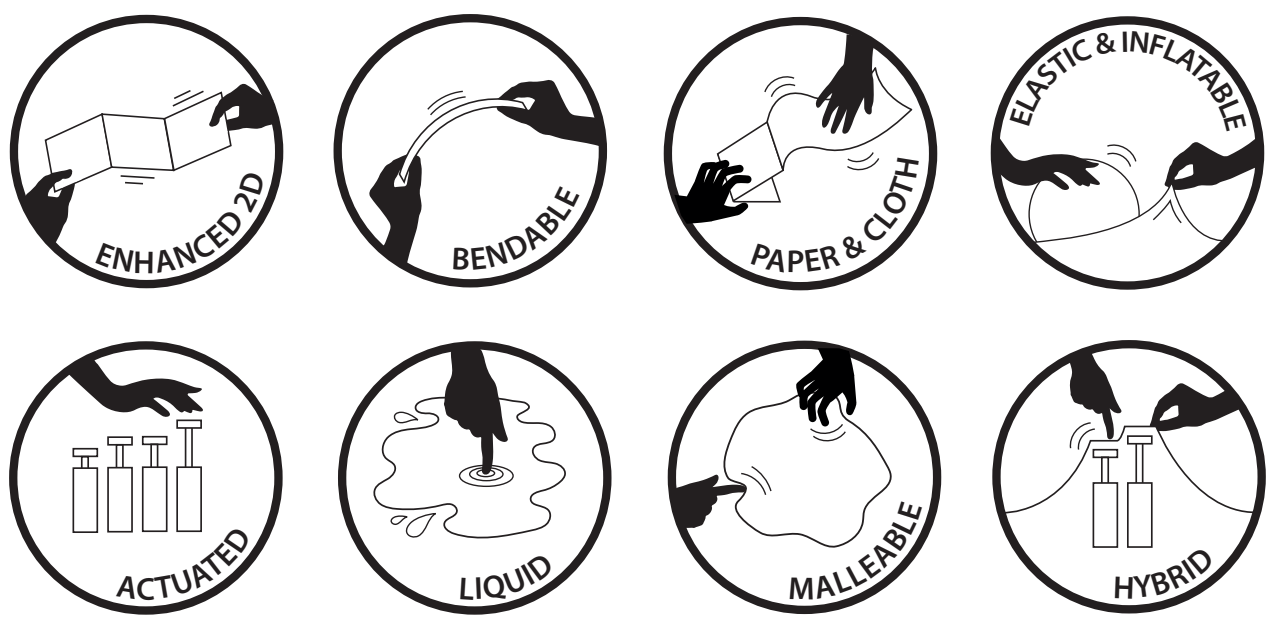

Fig. 3. Overview of shape-changing prototype categories

as input/output, but deformation is an input only (the user can manipulate the cloths' form, but the cloth cannot manipulate itself programmatically). Therefore it can be considered that bidirectionality is not a given, and as such not essential in the design of shape changing applications as meaningful interactions can be had across modalities.

4.3.2 Environmental compatibility/power requirement. Environmental compatibility (the suitability of a device for its environment [11]) and power requirement are important considerations for the future of shape changing devices, but at present are not included due to the prototypical nature of the examples - due to the immaturity of the field, these are future considerations. The application of shape change in real world scenarios must come before situational problem-solving at this stage.

4.3.3 Reversibility, transition quality \& accuracy. Of the remaining aspects of the hardware, reversibility is a given for shape change in this case, as otherwise there would be no form-based interaction past the initial deformation. Transition quality and accuracy are difficult to assume from the literature alone: without analysis of these aspects in particular for each prototype, we cannot begin to attribute these qualities to the mechanics of each device. The remaining dimensions (accuracy, trainability and complexity of actuation) are rooted firmly in the material/actuation type, and can be related directly back to the primary hardware categories.

\subsection{Interactive}

The interactive aspects of shape-change have been expanded from Figure 1 as these are the most important aspect of shape changing interfaces: without the user, a prototype is passive or remote [79]. Interaction is primarily defined by Rasmussen's initial review of shape-change and can be defined as direct, indirect and remote, this is discussed below. Interactive shape-changing art installations are included if they fulfil the earlier criteria (such as AegisHyposurface [26] or Protrude, Flow [47]).

The proximal considerations for the user are based on Rasmussen's classification of interaction (see previous paragraph), omitting only "none" as a type of interaction, for the reason given above. Direct proximity infers that the user can touch the surface of, or interact with the prototype directly 
(as with ClaytricSurface [84]), without the need for an additional item such as a ring or wand (as is the case with Linetic [48]). Indirect proximity requires an additional construct for the user to interact (such as a connected laptop as with Flexkit [32]) or the user can use mid-air gestures as a form of input - but this can exist in tandem with Direct proximity. This is also the case for Remote operation, which suggests that the interface can be controlled via infrared, wireless or Bluetooth technology, and therefore, in the case of wireless internet communication, from almost any distance.

Almost any kind of input or output could be designed for shape-changing interfaces, but the table records only current iterations. Smell, for example, has been used in clayodor [40] as an output, but this prototype is not included due to the separate nature of the input/output components. Future types of input might include those that are non-visible, such as radiation or air quality. Of the research surveyed, it can be seen that there is currently a greater variety of input than output. Inputs thus include: program (a program is used to control some aspect of the interface, such as the bend of the SMA [23], or visual imagery [71]); gesture [28, 48]; touch/haptic [68, 101]; light [89]; sound [26]; and deform (separate from simple touch sensitivity, this implies some force or movement is applied to change the shape of the available surface, whether it is bending [99], pushing [19] or more advanced deformation[84]).

Output is currently limited to: form (as discussed in relation to bi-directionality) e.g. [81, 104]; sound (deliberately generated, as opposed to an incidental sound generated by the mechanism) [49]; light (often as an artifact of projection [54], or internally generated [75]); and text/image [2, 59].

Number of users was also found to be relevant to interactions with prototypes - because it changes the way designers think about their interface - although it was not always explicitly written how many each device was designed for. Xpaaand [42] is a mobile device prototype based around one user perspective, but the discussion highlighted the possibility that a large change in width supports multiple user interaction. In comparison, inFORM/TRANSFORM's physical telepresence [53] is specifically designed to support remote interaction between two users. Aegis Hyposurface [26] is a large public installation, and therefore can support multiple users, hence it is listed as supporting all three user bases. Where number of users is not explicit, then the prototype user base is estimated based upon size: mobile phone devices are attributed to one user, tablet size devices to two users, and anything of tabletop size and above is seen to support multiple users.

\subsection{Temporal}

The notion of temporality in design is in its infancy, but is inextricably linked to both the physical and interactive dimensions of interaction for shape changing interfaces [102] (see Figure 1). Understanding the limitations of time and speed for each prototype is essential for implementing successful design strategies. Whilst categorizing existing work for Tables 1-8, the origin of control for speed was found to be important as it affects how interaction occurs and how the user experiences the prototype. Interfaces were found to support three types of control: program defined - the speed of change is defined by programming, as in Aegis Hyposurface [26] which can move up to $100 \mathrm{~km}$ an hour; material defined - limitations are placed on the speed with which a change can take place due to material constraints, such as with SMAs [80] or actuators [68]; and user definedthe user controls the speed of deformation via direct deformation at a chosen speed (but within the limits of the device) [48]; or all three [28].

Designing for temporality is at its most difficult when the potential exists on all three dimensions. The desire for speed from the user may not always match the intentions of the application - i.e. an educational application might move with deliberate sluggishness so the child cannot skip parts, or by increasing the speed of a transformation, essential information might be lost. The opposite is also true - when browsing a shape-library, you may need to skip ahead or traverse options swiftly. 
Table 1. Enhanced 2D prototypes comparison table based on the consolidated dimensions in Figure 2.

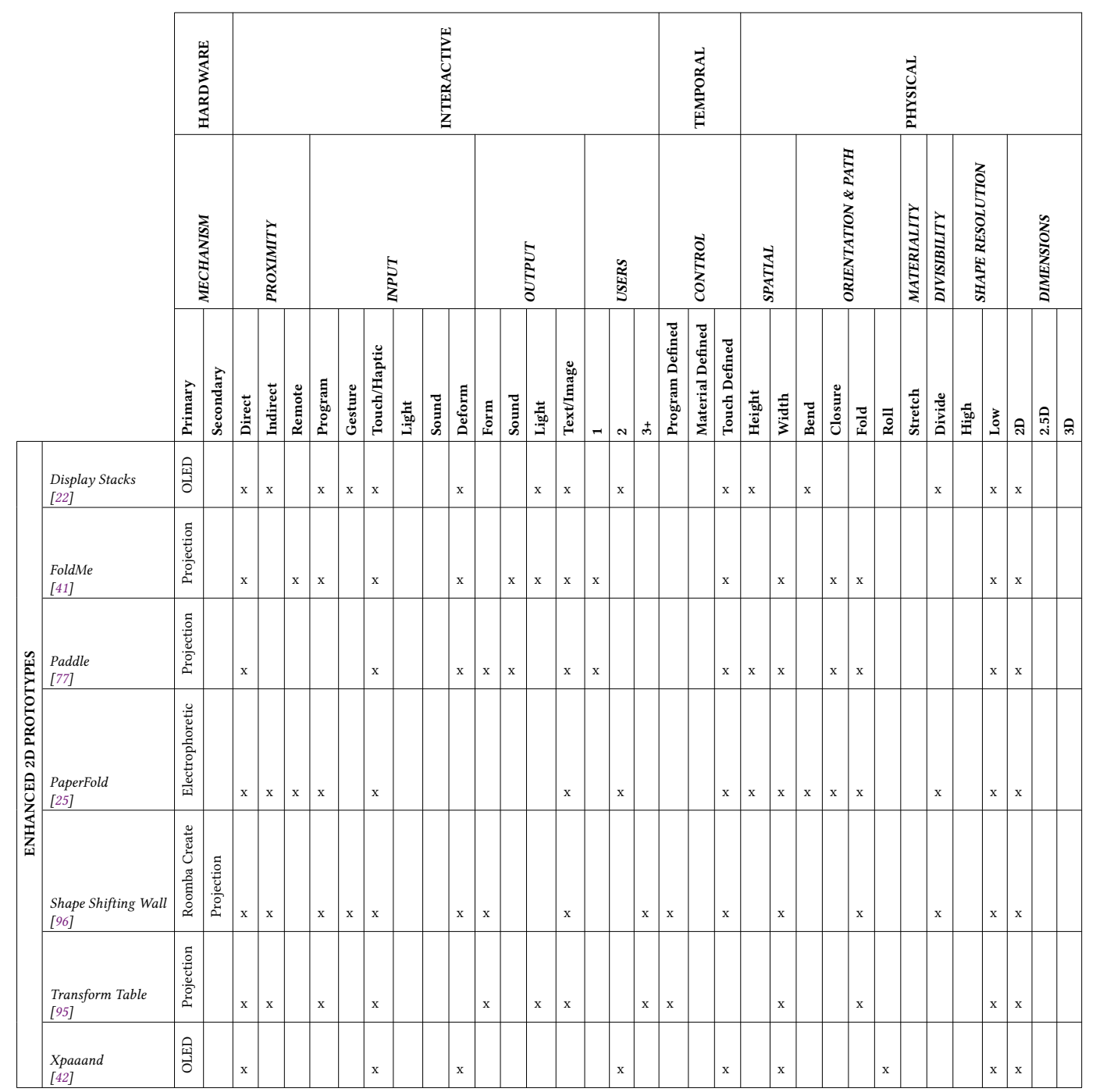

These aspects and more must be designed for, or against: the application must be able to control the pace that is most conducive for its purpose.

\subsection{Physical}

The physical characteristics of shape change emerge as quite distinct from the consolidated dimensions seen in Figure 1. Application of these dimensions to existing interface examples allows specific deformations to be noted and discussed. The physical changes of a surface range from the basic (height/width/bend) to the complex (closure/divide).

Height is the most commonly found change in actuated and material based deformations. It implies that the prototype experiences a change in height of the surface relative to its baseline (non-deformed starting point). This is always limited by the hardware making up the device. Height 
is also applied as a change to those prototypes which make use of one axis, in one direction [26] as the same idea applies despite the change in orientation.

Width, on the other hand, requires a two-way expansion across a plane, regardless of direction. This can be due to a stretch in the shape changing material from jamming for instance [72], or due to a device having the capability to be unfolded, such as with Paddle [77].

Bend, is most common with flexible displays such as Morphees [80] where the thickness of the OLED display or constraints of the SMA wires means that only a slight deformation of an otherwise $2 \mathrm{D}$ item is permissible.

Fold is closely related to closure - but the distinction lies between surface merely creasing and the surface folding entirely in on itself. Reabsorption happens in the cases where a ferrofluid is used ( $p B l o b[104])$ or edges meeting with a static surface (PaperFold [25]).

Roll also often goes hand in hand with highly flexible static surfaces - the best example being Xpaaand which is encased in rolls at either end [42].

Stretch is distinct from width, as it implies an area expansion from baseline based on materiality rather than simply displaying more of the same substrate. Stretchable materials are usually incidental hardware (like latex) and used over actuators [29] or in jamming [84].

Divide suggests either a modularity in actuators or components as seen in Hairlytop [68], PaperFold [25] and ShapeClip [28] or where a solution can be split into parts and reunited as in pBlob [104]. Shutters [10] is an interesting hybrid, using folds and splitting simultaneously to allow for a divided (or permeable) surface.

Resolution refers to shape-resolution as coined by Roudaut et al. [80] and incorporates the textural element as discussed earlier in section 3 [69]. A high shape resolution is the same as a high pixel resolution in that a 2 dimensional representation of a sphere on a low resolution screen would show "squaring off" or aliasing around the edges, whereas a low shape resolution sphere would have angular blocks making up its surface. Liquid interfaces have high shape resolution due to fact they do not rely on set sized nodes as actuators do.

Dimension falls between 2D and 3D, referring to 2.5D as either a limited 3D display (i.e. one plane of deformation only with projection as a separate construct) or as one where there is sufficient deformation possibility that the design-surface would need to allow for form if the display was to have an application design for it. 2D shape changing interfaces in this case are typically changing their area (width) but the design space is resolutely flat.

\section{CATEGORISATION AND ANALYSIS OF PROTOTYPES}

Following application of the previous consolidated dimensions to 84 existing prototypes, 8 distinct categories of prototypical device emerged based on the properties of the hardware and mechanism of the collected technologies. Physicality (hardware or primary mechanism) was the vital factor in assigning these categories as it had the most influence on user-interaction and shape-input/output. For example, a user interacts with an elastic interface in a very different way to an actuated interface (i.e. it is impossible to stretch a solid-state pneumatic pin).

The 8 categories are: Enhanced 2D (Table 1), Bendables (Table 2), Cloths \& Papers (Table 3), Elastics \& Inflatables (Table 4), Actuated (Table 5), Liquids (Table 6), Malleables (Table 7) and Hybrids (Table 8). These categories are clear groupings which stand out from a combined analysis, as they often share common themes not only within their hardware, but across the interactive, temporal and physical dimensions. A comparison between these categories can be seen in Tables 9 and 10 . Additionally, example photographs of prototypical devices within each category can be seen in Figure 2. Each category is discussed in detail below. 
Table 2. Bendable prototypes comparison table based on the consolidated dimensions in Figure 2.

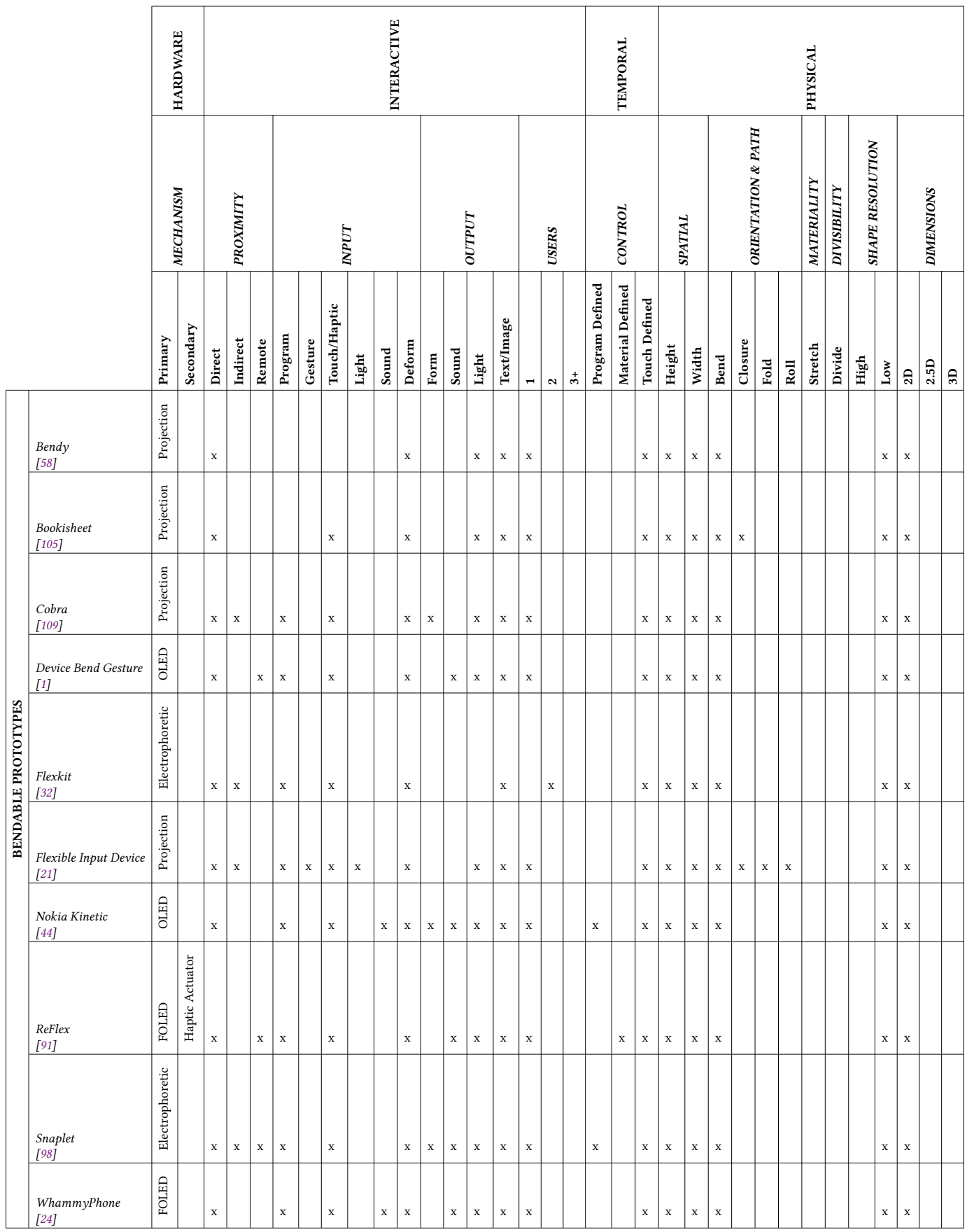


5.0.1 Enhanced 2D. Prototypes make use of multiple incidences of 2D screens which flex along either axis (see Table 1). Prototypes must have one or more screen or extra surface available which operates independently from its primary interface surface (see Figure $2.1 \&$ Figure 3 ). The primary method of shape-change is touch defined (with the exception of TransformTable [95]) Shape resolution is low.

These types of devices account for nearly $10 \%$ of the surveyed literature (7/84). Design for Enhanced $2 D$ interfaces should exploit multi-screen interactions or applications and either exploit or avoid the ensuing perceptual angles allowed by such prototypes (such as when a geometric shape such as a boat is constructed [23]). With regards to this, designers should also be a focus on user perception over more than 2 screens, as well as number of users and how they communicate about differing screen-states during multiple use interactions. Single user scenarios fit more commonly into existing device designs and therefore there are existing precedents (e.g. Nintendo $D S^{\mathrm{TM}}$ ).

5.0.2 Bendables. These devices have one display and interaction surface, but that surface has movement in terms of bend or flex at the corners, middle and edges (including twist) (Table 2). The image is essentially planar, and the shape-resolution low in comparison to the visual display, but the added emphasis on user interaction and programmed movement is how these prototypes differ from their Enhanced 2D counterparts. Design for Bendable interfaces is 2D single screen, with additional movement as its key feature - creating multiple modes of interaction.

Bendables account for just over $10 \%$ of the surveyed prototypes (10/84), largely focusing on either input and interaction [105] or physical, unobtrusive notifications [58]. Physical changes in shape to inform users of application states has links to the emotionality in shape-change, which has been explored in part by Rasmussen et al. [78, 79]. The prospect of anthropomorphising our user-interfaces adds a curious and exciting aspect to creating applications for shape-changing interfaces. Design for a Bendable also largely needs to focus on mapping interactions and outputs to the range of supported flexes for any given prototype (MorePhone supports 17 interactions [23]).

5.0.3 Papers \& Cloths. Table 3 shows prototypes which fulfil the criteria of Papers \& Cloths. These prototypes have one interaction surface, but are highly adaptive in terms of orientation and path, mimicking the characteristics of their non-interactive base-materials. Deformation is primarily user-controlled. These prototypes can borrow from web-design (in that re-flowable content to fit the visually available area is used) or be re-purposed into novel interface designs (wearables/furniture).

Around $16 \%$ of the prototypes in this summary are Papers \& Cloths (14/84). Devices of this nature would be beneficial in situations where they need to be portable, and condensed into small spaces for transport or covert use. For this reason they might be well-suited to multimedia applications where viewing size is important across a range of scenarios.

5.0.4 Elastics \& Inflatables. Elastics \& Inflatables are deformable interfaces that are made up of materials with built in stretch such as Elascreen [111]. Control here is shared between the actor (user) and the material (which has a high-speed return-to-baseline). These interfaces have an organic appeal (such as EmoBalloon [63]) but usually have limited shape resolution (with the exception of jamming interfaces [18]). Like Bendables, they can also exhibit emotional characteristics.

Just over $10 \%$ of shape-changing prototypes exhibit criteria which assign them to this category (9/84). Large scale elastic screens [100] suggest use cases such as exploration of data or gaming, whereas the organic nature of such interfaces makes them suitable for communication or tangible interaction with other users. A combination approach between jamming and larger elastic surfaces would yield more complex interaction styles and application opportunities. These pliable materials 
Table 3. Cloth and Paper prototypes comparison table based on the consolidated dimensions in Figure 2.

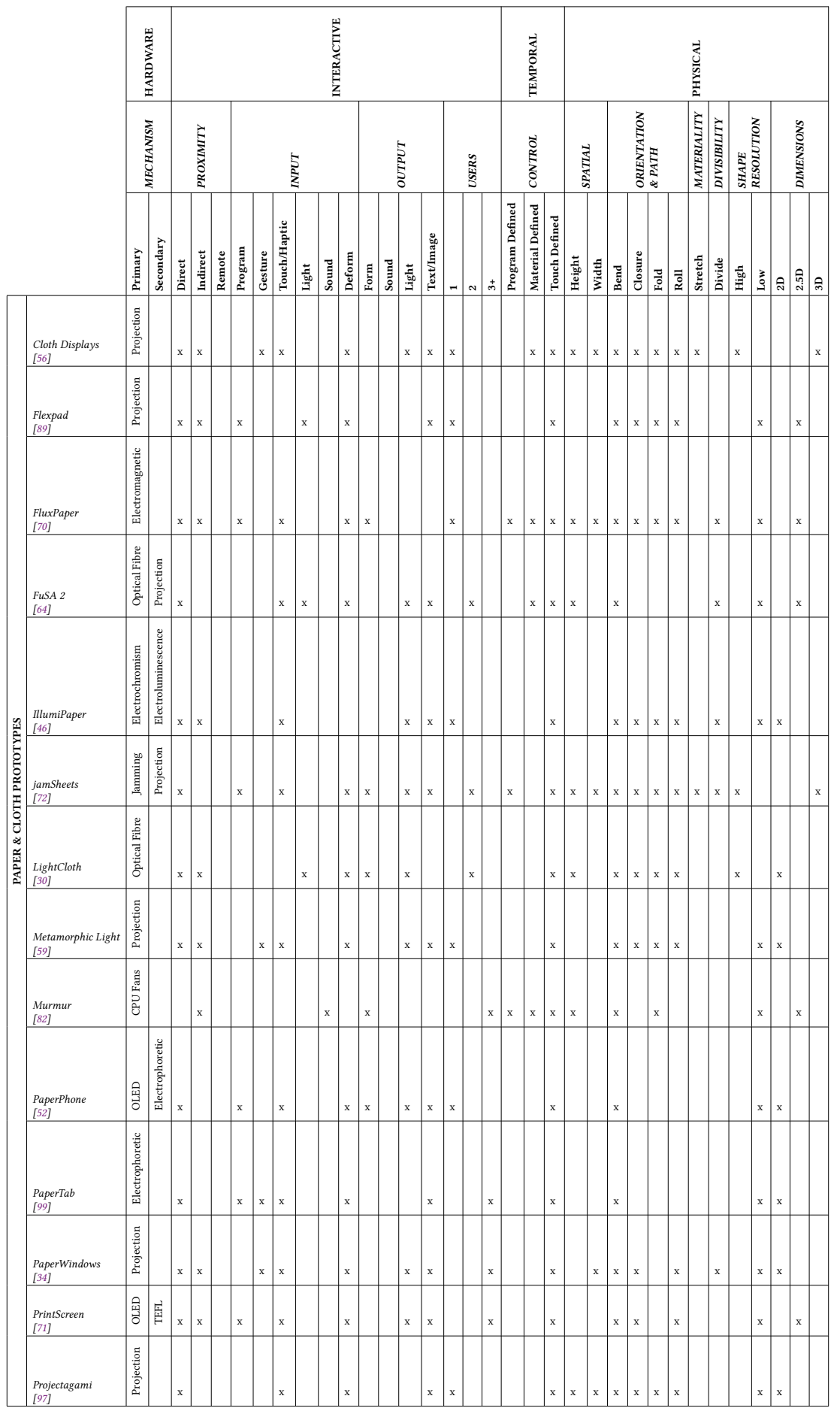


Table 4. Elastic and Inflatable prototypes comparison table based on the consolidated dimensions in Figure 2.

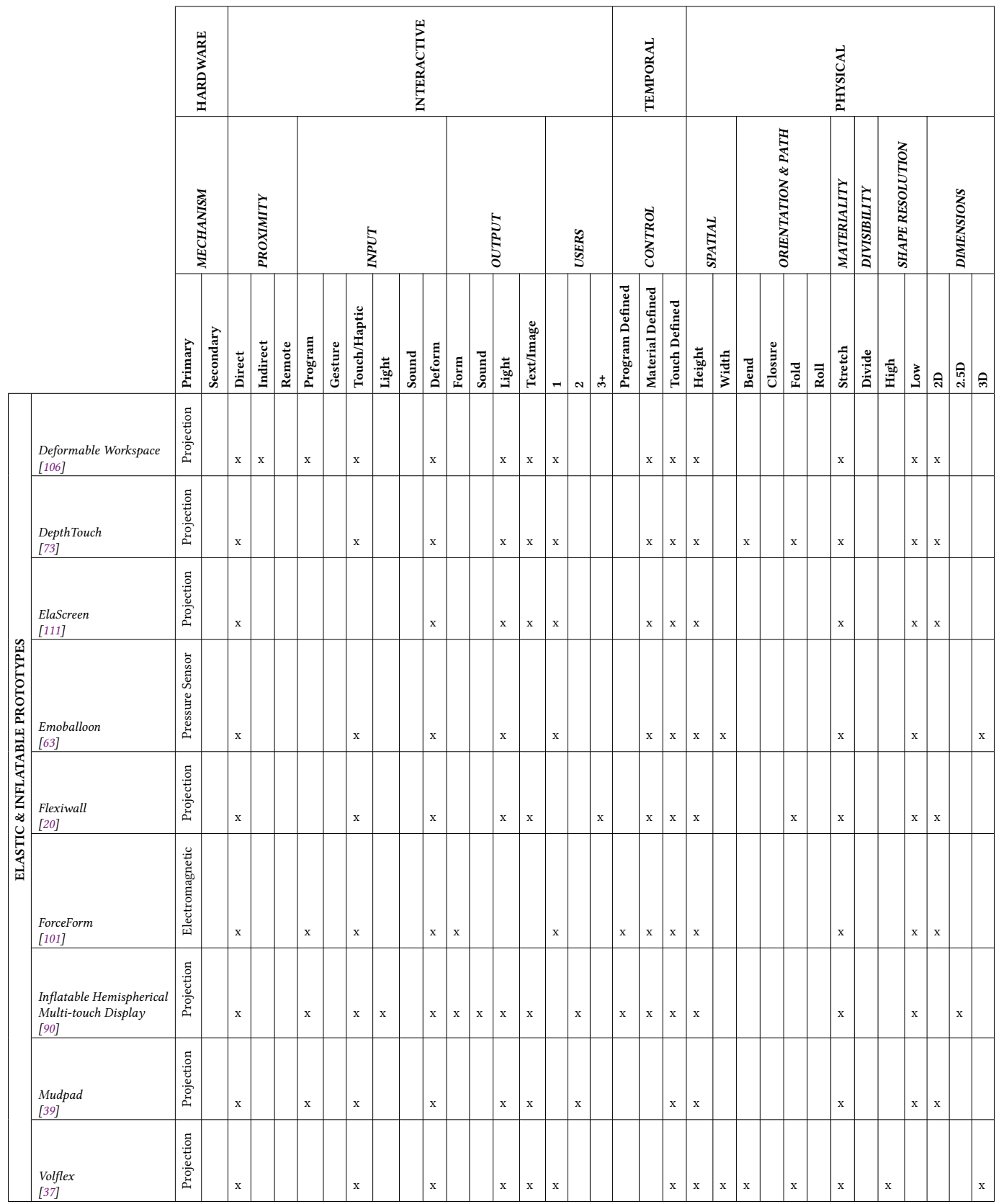

also have the potential to change their interaction area drastically, which would assist when multiple users need to collaborate on demand.

5.0.5 Actuated. Whilst the mechanics of each prototype are different, shape-change for these devices relies on separate mechanisms controlling each shape-pixel. Actuated interfaces are sometimes 
covered with a material substrate to create an undulating surface [26]. Some actuated prototypes have visual displays built-in. These prototypes usually have one repeated movement (bi-directional) and a limited height from baseline (flattened plane).

Actuated interfaces make up the largest proportion of shape-changing interface prototypes at just over a third of all those surveyed (29/84). This is likely because of the large variety of actuator types, outputs and ease with which each shape-pixel can be programmed to respond. As the largest grouping, Actuated interfaces are also the most diverse - supporting current applications which range from calm, environmental computing [8], to communicative architecture [10]. Researchers have already begun to think around the problem of shape-pixels for actuated interfaces by adapting an existing 3D programming language to allow for interaction and shape-change [107]. This is a vital step in giving other researchers and application designers the tools they need to build meaningful interactions for such devices.

5.0.6 Liquids. Liquid prototypes are complex and span between highly organic 3D shapes and viscose $2 \mathrm{D}$ shapes. Interaction is mainly indirect, although some substrates allow the user to touch the surface of the interface. Despite apparent limitless parameters, the current prototypes support only selected output (namely shapes, or sounds). To keep a liquid in a rigid state, one must exert continuous control, either via an indirect control device (such as a magnetic ring [48]) or via the programming of the control mechanism (usually electromagnetic).

Liquids account for the smallest number of single category prototypes in this area (5/84) - this is possibly due to the complexity of programming interactions and exerting control over such substrates. Despite this complexity, the potential in this area is unbounded. Potential focus might be on increasing direct interaction possibilities - such as through hybridisation with jamming [18].

5.0.7 Malleables. Malleables are clay-like or jamming substrates that afford the user a pliable, deformable surface with which to create high shape-resolution forms. Jamming does not take centre-stage here, as other materials have been used to create the same rigidity and control (e.g. Tunable Clay [18]). These prototypes have multi-dimensional input/output, but rely mainly on projection to supply equally high resolution graphics.

Malleable interfaces also represent only a small number of the surveyed technologies at under $10 \%$ (7/78). Despite having high shape-resolution, the reliance on projection for visualising complex graphics means that these devices are not, as of yet, portable. In their current state, they are best suited to permanent installations or interactive multiple-user scenarios.

5.0.8 Hybrids. Hybrid interfaces are relatively new in the field, and combine two (with the potential for more) of the former categories to create the interaction surface. This suggests that this category has more of an overarching nature, and could be addressed as such, however, given the limited data we have on these they are shown as a final, complex category. Both TableHop [83], Obake [13] and the second iteration of Mephistophone [31] combine an actuated base with an elastic surface to create additional methods for user interaction. This layering up of mechanisms is reminiscent of Seah et al's [87] space-suit glove prototype which enables those in sealed suits to experience physical textures - however, much attention has been given in the three hybrid prototypes to the complexity of interaction between layers and in combination. Table 8 shows an overview the current Hybrid interfaces.

Although some other of the included prototypes already make use of some materials from other categories (eg. Projection is used across the board), these prototypes do not fully support the features of both categories at present, whereas the hybrid examples given here enable users to make use of both types of interaction on the same surface. Hybrids are relatively rare in the study of shape-changing interfaces (3/84) but are likely to form part of the next stages of research. 
Table 5. Actuated prototypes comparison table based on the consolidated dimensions in Figure 2.

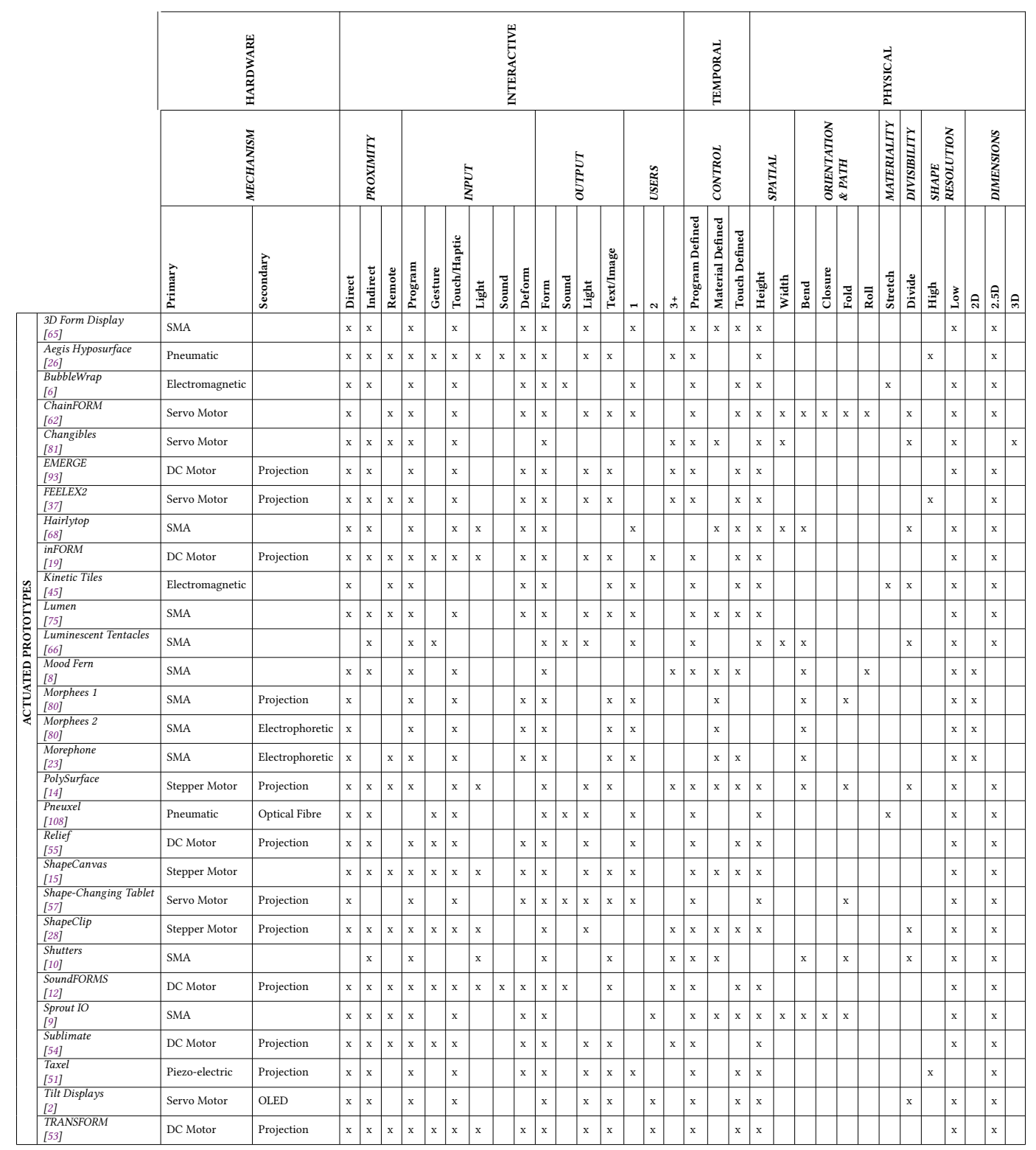

The implications for application design for hybrids are that the interaction possibilities become extremely complex, cross different modalities, temporalities, and can support multiple users in each - potentially at the same time. The potential for mismatch, both interactive and perceptual, is such that the possibilities also become a limiting factor. 
Table 6. Liquid prototypes comparison table based on the consolidated dimensions in Figure 2.

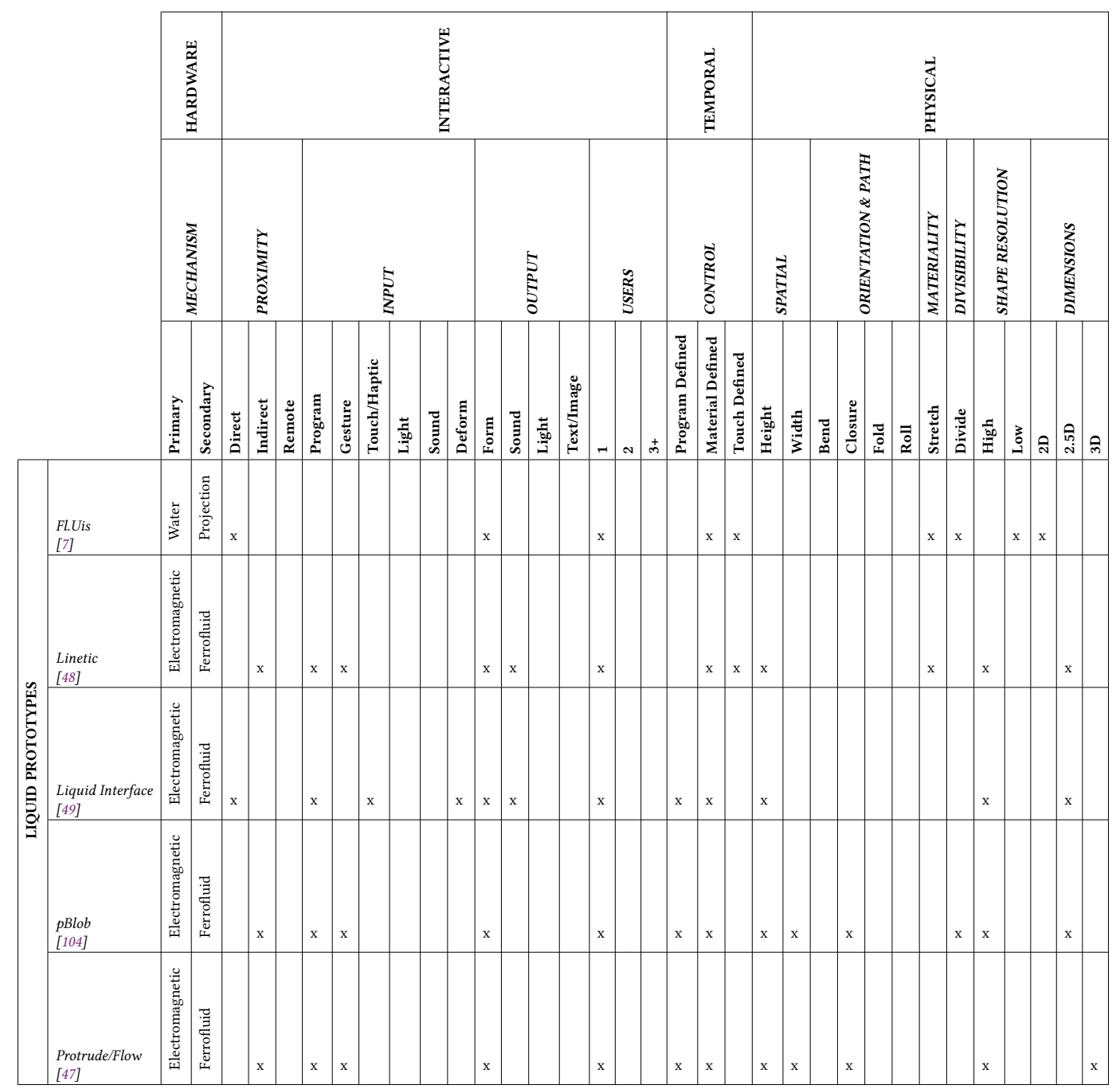

\subsection{Categorisation Summary}

The current state-of-the-art is largely represented by this categorisation of shape-changing interfaces (Tables 1-8). The field as a whole however, is constantly evolving - and there may be additions or whole new categories within a relatively short space of time. Each interface category has its strengths and weaknesses, and these are continually evolving, making designing for such structures an iterative process. Many research papers suggest future design modifications for their existing prototypes, and it is these which enrich and take the field forward.

A summary diagram of the prototype categories can be seen in Figure 3, whereas an analysis of feature frequency across all 8 categories can be seen in Table 9. Table 10 provides an overview of the limitations \& current uses for each prototype category. The overall comparison of features between categories (Table 9) produces some additional findings which offer another perspective to the analysis contained here. These are discussed below. 
Table 7. Malleable prototypes comparison table based on the consolidated dimensions in Figure 2.

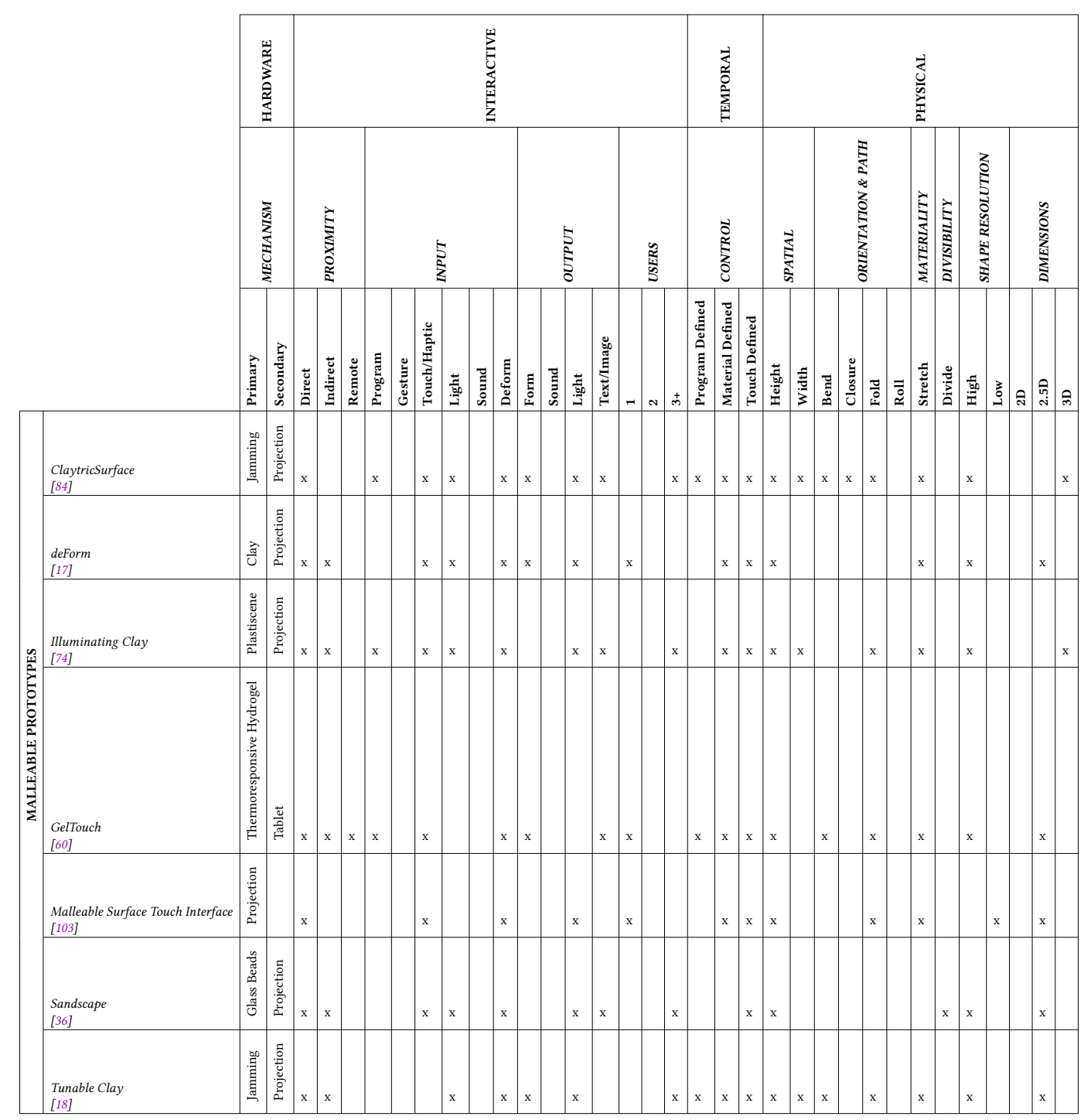

5.1.1 The Problem with Projection. Over half of all prototypes included in this dataset rely on one form or another of projection, e.g. backlit as with TableHop [83] or, more commonly, top-lit as is the case with Metamorphic Light [59]. The overuse of projection to achieve detailed imagery or interaction shows that there is a need to put more resources into embedded displays and shows the immaturity of the field in that respect - or that there is a need for advanced materials that have not yet been developed, or are currently being developed, such as Yokota et al.'s Ultraflexible organic photonic skin [110]. Embedding high quality displays into shape-changing devices would create a seamless user-experience that is lacking in current prototypes, enhancing the notion of the phygital (combining physical and digital into one): Projection is a useful tool for rapid prototyping, but it presents an interrupted user experience when top-lit (occlusion from hands/objects), and 
Table 8. Hybrid prototypes comparison table based on the consolidated dimensions in Figure 2.

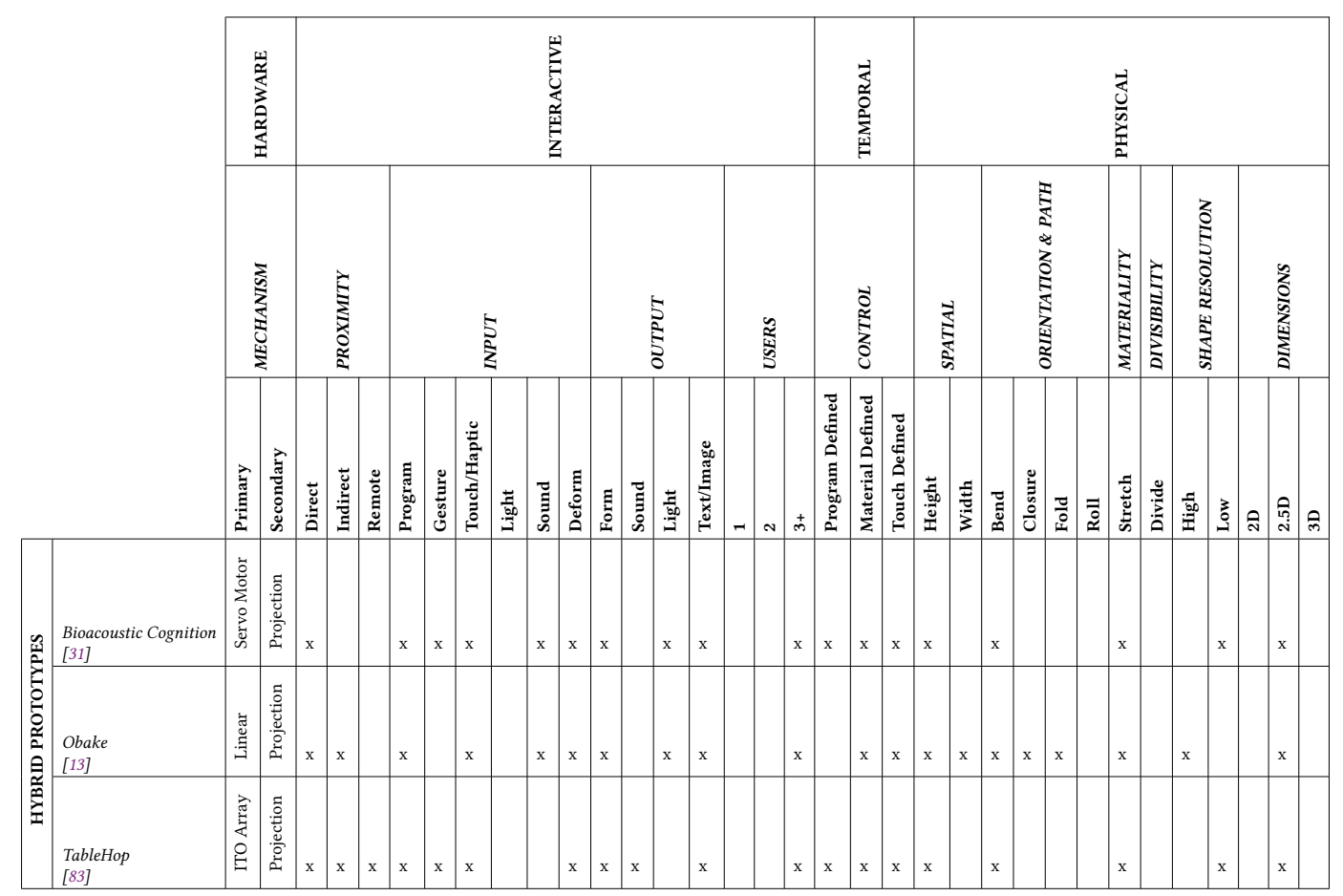

makes prototypes bulky and difficult to transport both when top and bottom-lit - meaning it is more difficult to get these devices out of the laboratory for meaningful testing, and that they will be unlikely to go into commercial development and production in their current form.

5.1.2 Multi-dimensional Change. Hardware and mechanism limitations can also effect the interactive qualities of devices. Poupyrev's notion of $R G B H$ shape-pixels [76] reflects the current state of play, but does not leave space for the exploration of multi-dimensional change. As an example, actuated interfaces can always display height, but very rarely does this combine with the type of shape-change in the orientation/path category. To attempt to expand on the interactions available to this subset of interfaces, combining the properties of a paper or cloth interface with the mechanised movement of an actuated interface would give rise to some novel data, e.g. using paper-style creases alongside the fluidity of cloth, with the rigidity and movement of actuated shape-pixels.

5.1.3 Number of Users. User data across the categories shows that just over half of all the prototypes analysed are developed for single users, although there is still a significant number which do support 3 or more users. This is likely in most cases to be a constraint of size, lack of divisibility, or difficulties in enabling multiple users to interact on the same surface. Collaborative usage and shape-changing interaction on these interfaces has not yet been well documented, and relates to the complexities of perception which are discussed later.

5.1.4 Control. With regards to temporality and control, there is a tendency for the user to be the primary locus of control of speed for most prototypes (around 84\%), e.g. with Bendable or Enhanced $2 D$ where the mechanism does not deform without user input (although some of 
the $84 \%$ also support multiply defined methods of control). The reasoning behind this could be that the user exerts primary control over shape-change merely because the materials used in such prototypes are not yet complex (e.g. paper or elastic rather than integrated hybrid forms with progammable actuated components) - however, given the importance of the user in any advancement in interaction design for shape-change, focusing on retaining the user as the primary factor in temporal control should be important to researchers.

\section{DISCUSSION}

The story of shape change so far is one of prototyping within existing technological constraints. By creating content for that which we have now, we will be able to lay the groundwork for a future shape-changing application design. With Ishii's [35] vision-driven design we look to the future, but this can happen only when we have truly understood the present. Whereas Kwak's framework [50] supports design engagement for shape change via tangible models, it is not based upon contemporary research prototypes. In contrast, Ishii works around existing technology to speculate as to the future of shape-changing interfaces. It is from/with Kwak's explorations and toward Ishii's speculation into which this paper places itself.

\subsection{Supporting Application Design}

The categorisations supplied in this paper break down the current state-of-the-art into clear boundaries. Therefore, a designer making an application for any existing interface will be able to look to the associated attributes and supported features, and sketch an outline for what must be considered during the iterative design process. For an actuated interface, for example, one must consider how many shape-pixels are available, the speed with which these are required to move to communicate the application's intent, the level of visual detail supported, and so on.

To elaborate, for those wishing to apply the framework in context of interface design, it is suggested that those using the classification query the intended outcome of the research - for example - What is the desired interaction in mind - and therefore what type of actuation best suits this outcome? A study wishing to analyse latency on moving pins would almost certainly need bi-directional actuators, whereas a study examining calm computing and peripheral shape-change might wish to examine the biological movements of natto cells or SMAs. Alternatively, if there is a platform in mind but not the knowledge of the types of user interaction required to enable user-testing then the researcher might look at number of users and the types of input and output supported.

The taxonomy can be interrogated in varying degrees depending on the nature of the research, although it should also be noted that there is a "trade off" between different types of shape-changing interface, which is another factor that can be easily seen from the available data. To provide an example in context of the latter, if you require an approximation of natural movement then you would almost certainly use natto cells or SMAs in lieu of servo motors. Another example of "trading off" could be choosing between hardware types within one category - i.e. if you require the portability of shape-clips but the advanced material properties of Transform (recently examined by [62]) you will need to decide which property is more salient for the research at hand.

Essentially, this paper is a library of shape-change, and can be queried as such: for any of the currently available shape-changing prototypes, a designer can now pick out the key features and limitations. It is hoped that this could open up multi-disciplinary collaborations, as well as those within the field. Although the question is raised: Is it the applications that will drive the technology or the technology that will drive or limit the design of applications? 
Table 9. Category summary of Prototypical Shape-Changing Interfaces showing totals across the consolidated dimensions.

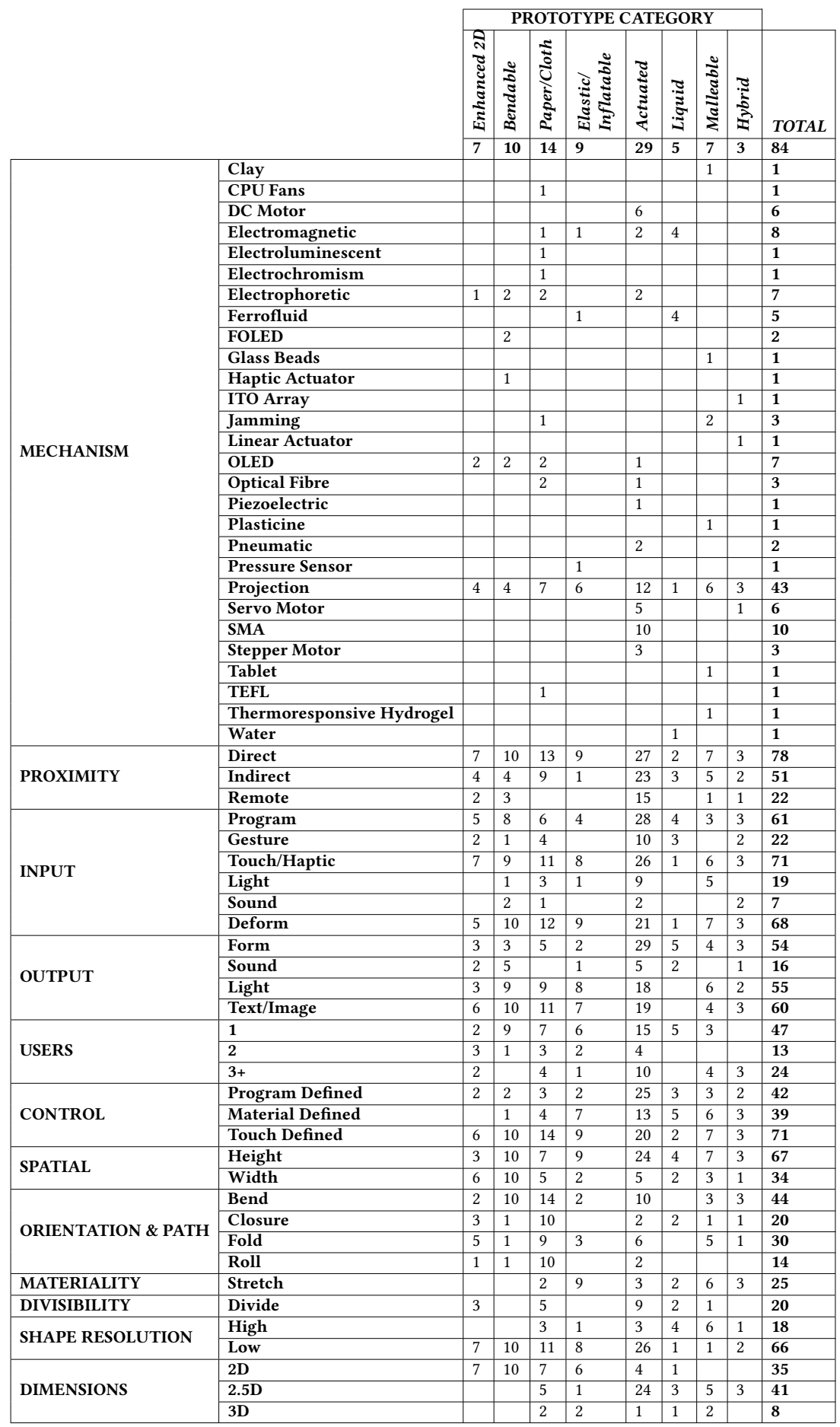




\subsection{Limitation in Design}

A successful multi-dimensional application designer must not only design for the capacities of shape change but against the limitations imposed by the hardware of the device (it must conform or have constraints [35]). These limiters actually reign in the design space, and offer a firm ground from which to work backwards from. A future where devices have unlimited dimensional potential (such as Ishii's Perfect Red [35]) must be built up to, working toward a theory of content on the lower fidelity devices first. Limitations are not merely device specific however, they can be built in as the program requires - working as areas of rigidity or non-interaction, like the background of a website around a clickable link. The challenge of programmed rigidity is not only one of hardware, but also of temporality - how quickly a force limiter is made or released can affect the users' experience of an application, not to mention interface safety. An exception to rigidity might be for a free-form sculpting application. Hardware limiters include (but are not exclusive to) the following:

Distance from baseline: Several studies state the total usable height [28] or width of their device [42]. For material based interactions, total distance from baseline must be calculated from the maximum stretch or available slack of the surface at one point at any given time.

Shape-resolution: As discussed in the previous section, deformation limiters are based on the type of device for which the application is being designed for. The lower shape resolution but highly interactive devices have narrow limits in comparison to the high resolution liquid shape displays.

Image resolution: Based on the image resolution of the device - a block-pixel image will afford a narrow design space with which to work with, whereas a projected, high resolution image will interact in multiple ways with areas of height and deformation, and present a challenge for users utilizing multiple viewing angles [76].

Stretch: An important consideration when designing for areas of rigidity: rigid areas may limit the deformation of surrounding interaction zones. Stretch ensures that deformation is still possible between closely spaced rigid objects.

Temporality: Speed of change is sometimes limited by the hardware (such as with motorized actuators), and so will need to be built into design considerations. Maximum and minimum speeds for deformation should be made available to the designer, or tested prior to finalizing applications.

Holman [32] mentions the current limitations of readily available electrophoretic displays (less than $1 \mathrm{fps}$ ) and how designing for such device displays requires advanced programming knowledge. If this knowledge is lacking, the rapid development of applications will suffer. This supports research in which those in the arts are encouraged to learn to code [88] and vice versa [16]. This new space of shape changing interaction design requires a new generation of multi-skilled designer-makers it is possibly not enough to simply be a competent developer or designer when new technology stretches the limits of imagination.

\subsection{Future Use Cases}

Application of shape-changing prototypes is so far mostly limited to improving items we already have in 2D such as phones, tablets and worktops. Those prototypes looking at shape construction begin to imply a new way of using form and interaction, however, user-driven research is needed to identify new types of interactive shape-changing product where need or desire exists. Following Bannon's call for a more "human-centred perspective" on HCI, Sturdee et al. carried out a study using a participant pool taken from the general public [92] and found that a range of shapechanging products were desired or suggested - not limited to, but including, interfaces, architecture, landscapes and wearables. 
Table 10. Summary of the features, limitations and current use cases of Prototypical Shape-changing Interfaces.

\begin{tabular}{llll}
\hline Prototype & Primary Feature & Limitation & Current Use Cases \\
\hline Enhanced 2D & Multi-screen & Inflexible & Phone /Tablet \\
Bendable & User-Interaction & Low Shape-Resolution & Phone /Tablet \\
Paper/Cloth & Orientation & User-Defined Temporality & Phone /Tablet/Workspace \\
Elastic/Inflatable & Stretch/Emotionality & Material-Defined Temporality & Emotional Communication/Workspace \\
Actuated & Bi-Directionality & Low Shape-Resolution & Physical Telepresence/Wrapped Interfaces \\
Liquid & High-Malleability & Low User-Control & Artistic Installation \\
Malleable & High Shape-Resolution & Projection-based Graphics & Workspace \\
Hybrid & Complex Interaction & No full 3D version & Information Visualisation \\
\hline
\end{tabular}

As the field develops, we may need to re-imagine the interface as something beyond the tablets and mobile-phones that we use today. Wearables and Internet-of-Things technology bring connectivity to the familiar and often mundane, whereas adaptive architecture (e.g. Schnadelbach's ExoBuilding [86]) turns our living space into an opportunity for interaction. Within shape-change, BubbleWrap [6] looks toward creating a technology that can be wrapped around anything to create an on-demand interface. This is not the only example of future-use cases being highlighted in papers - others suggest the next iteration of their device as they write up the first, and some, like Ishii [35] employ design fictions to envisage the future. It is because of this that it is likely that interaction-driven rather than device-driven application design is likely to take priority in the future, and hence developing user-experience design for this field is an important step.

\subsection{User-Experience and Emotionality}

User-centred design is a mature field and well applied in designing current interfaces and applications, but is only just beginning to take the fore in shape-change literature. Most shape-changing prototypes are highly tangible, and usually support multi-sensory input or output. This means that the user must learn a new set of skills to interact with such technologies, alongside their existing knowledge. The prototypes discussed here also have the added factor of emotionality, that is, that movement and shape-change can create an affective response. Deployment "in the wild" of shape-changing devices has been studied, (such as in the Shape-Changing Bench [78]), and it is Rasmussen who is attempting to bring focus onto user-experience in this field. To successfully create applications for these "magical" devices, designer, researcher and user must collaborate in first developing a novel practice of user-experience.

\subsection{Perception}

Few researchers make the connection between actuation, and altered perceptive state. Poupyrev, however [76] mentions that differing viewing angles will alter the experience, suggesting user mobility and/or display adaptation as a solution, touching briefly upon the idea that the display could alter to make perception easier from multiple locations, which also relates back to optical illusions (such as distorted advertising blocks on football pitches which appear square when seen from a remote camera).

The distinction is also made between asynchronous and synchronous states (i.e. graphics/shape mismatch), creating yet another dimension for the viewer to interpret, and the designer to create. This links into design prototyping where there is a distinction between "looks like" and "works like". Fidelity in either one of these areas affects possible interactions and thus the overall look and feel of an application design. 


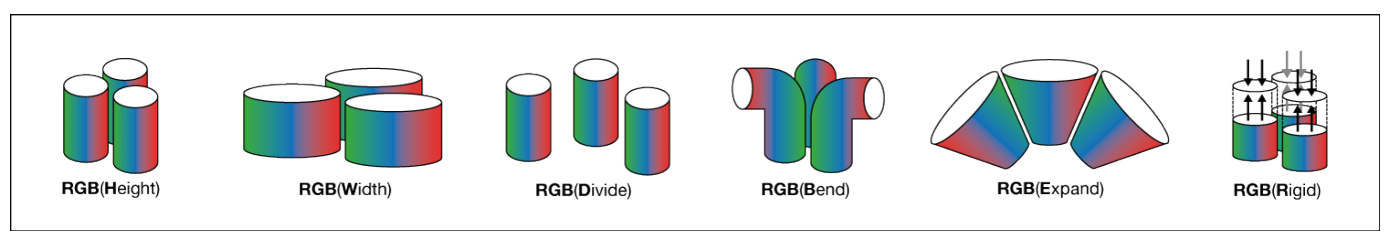

Fig. 4. Possible development of Shape-Pixel states based on RGB-H principle [76]

\subsection{Ethical Considerations}

If our computers become tangible, we open up ourselves to the notion of unwanted tangible interaction, perhaps unbidden, in the case of 3D spam [61]. Chat rooms become a step more dangerous for our children, as the unknown quantity of remote touch becomes possible. Control thus becomes more important - if AegisHyposurface [26] can move at speeds of 100kph, how can we design to prevent injury? Can closure of a surface cause trapped fingers - will there be a safety cut off? This extra concern must be incorporated into design - physical safety adds an extra dimension of concern for designers - something that is not currently needed for 2D displays.

\subsection{Future work}

The field of shape-changing interface prototypes as it currently stands is outlined in detail in this paper. At the time of writing, researchers are already beginning to combine mechanisms and interactions between prototypes to create hybridised interfaces [13, 31, 83]. This suggests that a logical step forward for some researchers would be to combine the characteristics between other current prototypes to create high-fidelity and multiple-interaction supporting shape-changing interfaces.

Hybrids are capable of both sets of interactions, and thus present a more complex design space that must be built from the specifications of the component hardware. Future shape-changing interfaces are likely to incorporate even more aspects of the prototypes seen here, and whereas interaction and applications can be anticipated from the design for their predecessors to some extent, the design space where all interactions are possible registers and even more complex problem to users, researchers and designers. It is hoped that this categorisation of existing prototypes might prompt collaborative work between referenced groups to create such hybrids, and also bring designers on board to test their application potential.

Poupyrev discusses the notion of RGBH graphics, where colour information is as we expect to find in GUIs, but with pixel height as an added numerical component [76]. Although a logical step for actuated displays, for a shape-changing display to be truly malleable, it must not only move on one axis, but several - turning corners, expanding or folding into itself. It would therefore make sense to use the RGB-H space, but replace ' $\mathrm{H}$ ' with $n$, where $n$ represents a different dimensional change in shape pixel state (see Figure 4 for examples of possible iterations based upon RGB-H). This idea of advanced shape-pixels is far from being realized, but could be expanded on via further reasearch.

The community surrounding these advances is often a highly specialized base of researchers and students, and as such user testing and the resulting inferences might be biased. Bannon [5] mentions that the 'human' side of HCI has been lacking in recent years, and Rasmussen [79] calls for more "high quality data" on user experience for shape change. By eliciting input from non-expert users, we might realize new directions for shape change, and nurture the design space. Finally, it is anticipated that the categorisation of shape-changing prototypes will be added to as the field 
moves forward in coming years, and thus there will more complex aspects for designers to consider, alongside the implications for the user.

\section{CONCLUSIONS}

This paper has consolidated multiple reviews for shape-change, mapped existing prototypes onto this framework, and suggested 8 categories for different types of shape-changing interface based on the hardware used and the limitations/opportunities provided by such devices. These categories have been further reviewed in relation to application design for GTUIs and guidelines suggested to make the first steps toward a standardised future practice. The analysis and classification of shapechanging interfaces will be an ongoing task as these technologies develop, but it is hoped that this review of the field will enable designers to make decisions about designing for these devices, and carry out user studies relevant to specific applications and hardware. It is also hoped that creating transparency in the field might elicit new collaborations and prompt interdisciplinary research, as there are many opportunities. Future iterations should include investigation of non-standard interface technologies, detailed user-experience analysis and collaborative practice to inform a new paradigm of user-experience design, and sample application design based on new guidelines following on from developing the categorisations shown here.

\section{ACKNOWLEDGMENTS}

This research was supported by HighWire Doctoral Training Centre at Lancaster University funded by the RCUK Digital Economy Programme through the EPSRC (Grant Reference EP/G037582/1), and partially-supported by GHOST, a project funded by the European Commission's 7th Framework Programme, FET-Open scheme (Grant \#309191).

\section{REFERENCES}

[1] Teemu T Ahmaniemi, Johan Kildal, and Merja Haveri. 2014. What is a device bend gesture really good for?. In Proceedings of the 32nd annual ACM conference on Human factors in computing systems (CHI '14). ACM, 3503-3512.

[2] Jason Alexander, Andrés Lucero, and Sriram Subramanian. 2012. Tilt displays: designing display surfaces with multi-axis tilting and actuation. In Proceedings of the 14th international conference on Human-computer interaction with mobile devices and services (MOBILE HCI '12). ACM, 161-170.

[3] Tobias Alrøe, Jonas Grann, Erik Grönvall, Marianne Graves Petersen, and Jesper L Rasmussen. 2012. Aerial tunes: exploring interaction qualities of mid-air displays. In Proceedings of the 7th Nordic Conference on Human-Computer Interaction: Making Sense Through Design (NordChi '12). ACM, 514-523.

[4] Mahoro Anabuki and Hiroshi Ishii. 2007. AR-Jig: a handheld tangible user interface for modification of 3D digital form via 2D physical curve. In 6th IEEE and ACM International Symposium on Mixed and Augmented Reality, 2007 (ISMAR '07). IEEE, 55-66.

[5] Liam Bannon. 2011. Reimagining HCI: toward a more human-centered perspective. Interactions 18, 4 (2011), $50-57$.

[6] Olivier Bau, Uros Petrevski, and Wendy Mackay. 2009. BubbleWrap: a textile-based electromagnetic haptic display. In CHI '09 Extended Abstracts on Human Factors in Computing Systems. ACM, 3607-3612.

[7] Tim Campbell, Cesar Torres, and Eric Paulos. 2015. Fl. UIs: Liquid-Mediated Vision Based Touch Surfaces. In Proceedings of the Ninth International Conference on Tangible, Embedded, and Embodied Interaction (TEI '2015). ACM, $85-88$.

[8] Bernard Cheng, Antonio Gomes, Paul Strohmeier, and Roel Vertegaal. 2014. Mood fern: exploring shape transformations in reactive environments. In Proceedings of the 11th Conference on Advances in Computer Entertainment Technology (ACE '14). ACM, 60.

[9] Marcelo Coelho and Pattie Maes. 2008. Sprout I/O: a texturally rich interface. In Proceedings of the 2nd international conference on Tangible and embedded interaction (TEI '08). ACM, 221-222.

[10] Marcelo Coelho and Pattie Maes. 2009. Shutters: a permeable surface for environmental control and communication. In Proceedings of the 3rd International Conference on Tangible and Embedded Interaction (TEI '09). ACM, 13-18.

[11] Marcelo Coelho and Jamie Zigelbaum. 2011. Shape-changing interfaces. Personal and Ubiquitous Computing 15, 2 (2011), 161-173. 
[12] Aubrey Colter, Patlapa Davivongsa, Donald Derek Haddad, Halla Moore, Brian Tice, and Hiroshi Ishii. 2016. SoundFORMS: Manipulating Sound Through Touch. In Proceedings of the 2016 CHI Conference Extended Abstracts on Human Factors in Computing Systems. ACM, 2425-2430.

[13] Dhairya Dand and Robert Hemsley. 2013. Obake: interactions on a 2.5 D elastic display. In Proceedings of the adjunct publication of the 26th annual ACM symposium on User interface software and technology (UIST '13). ACM, 109-110.

[14] Aluna Everitt and Jason Alexander. 2017. PolySurface: A Design Approach for Rapid Prototyping of Shape-Changing Displays Using Semi-Solid Surfaces. In Proceedings of the 2017 Conference on Designing Interactive Systems. ACM, 1283-1294.

[15] Aluna Everitt, Faisal Taher, and Jason Alexander. 2016. ShapeCanvas: An Exploration of Shape-Changing Content Generation by Members of the Public. In Proceedings of the 2016 CHI Conference on Human Factors in Computing Systems. ACM, 2778-2782.

[16] Paul Fishwick. 2003. Nurturing next-generation computer scientists. Computer 36, 12 (2003), 132-134.

[17] Sean Follmer, Micah Johnson, Edward Adelson, and Hiroshi Ishii. 2011. deForm: an interactive malleable surface for capturing 2.5 D arbitrary objects, tools and touch. In Proceedings of the 24th annual ACM symposium on User interface software and technology (UIST '11). ACM, 527-536.

[18] Sean Follmer, Daniel Leithinger, Alex Olwal, Nadia Cheng, and Hiroshi Ishii. 2012. Jamming user interfaces: programmable particle stiffness and sensing for malleable and shape-changing devices. In Proceedings of the 25th annual ACM symposium on User interface software and technology (UIST '12). ACM, 519-528.

[19] Sean Follmer, Daniel Leithinger, Alex Olwal, Akimitsu Hogge, and Hiroshi Ishii. 2013. inFORM: dynamic physical affordances and constraints through shape and object actuation. In Proceedings of the 26th annual ACM symposium on User interface software and technology (UIST'13). ACM, 417-426.

[20] Ingmar S Franke, Mathias Müller, Thomas Gründer, and Rainer Groh. 2014. FlexiWall: Interaction in-between 2D and 3D Interfaces. In HCI International 2014-PostersâĂŹ Extended Abstracts. Springer, 415-420.

[21] David T Gallant, Andrew G Seniuk, and Roel Vertegaal. 2008. Towards more paper-like input: flexible input devices for foldable interaction styles. In Proceedings of the 21st annual ACM symposium on User interface software and technology (UIST '08). ACM, 283-286.

[22] Audrey Girouard, Aneesh Tarun, and Roel Vertegaal. 2012. DisplayStacks: interaction techniques for stacks of flexible thin-film displays. In Proceedings of the SIGCHI Conference on Human Factors in Computing Systems (CHI '12). ACM, 2431-2440.

[23] Antonio Gomes, Andrea Nesbitt, and Roel Vertegaal. 2013. MorePhone: a study of actuated shape deformations for flexible thin-film smartphone notifications. In Proceedings of the SIGCHI Conference on Human Factors in Computing Systems (CHI '13). ACM, 583-592.

[24] Antonio Gomes, Lahiru Priyadarshana, Juan Pablo Carrascal, and Roel Vertegaal. 2016. WhammyPhone: Exploring Tangible Audio Manipulation Using Bend Input on a Flexible Smartphone. In Proceedings of the 29th Annual Symposium on User Interface Software and Technology. ACM, 159-161.

[25] Antonio Gomes and Roel Vertegaal. 2015. PaperFold: evaluating shape changes for viewport transformations in foldable thin-film display devices. In Proceedings of the Ninth International Conference on Tangible, Embedded, and Embodied Interaction (TEI '15). ACM, 153-160.

[26] Mark Goulthorpe, Mark Burry, and Grant Dunlop. 2001. Aegis hyposurface: The bordering of university and practice. In Proceedings of the 21st Association for Computer Aided Design in Architecture (ACADIA '01). 344-349.

[27] Wenjun Guo, Sang-Ung Yi, Minkyu Choi, Seungha Yoo, and Kyungwon Lee. 2013. Garden agua: three-dimensional tangible display enabled by arranged water jet. In SIGGRAPH Asia 2013 Emerging Technologies. ACM, 9.

[28] John Hardy, Christian Weichel, Faisal Taher, John Vidler, and Jason Alexander. 2015. ShapeClip: towards rapid prototyping with shape-changing displays for designers. In Proceedings of the 33rd Annual ACM Conference on Human Factors in Computing Systems (CHI '15). ACM, 19-28.

[29] Chris Harrison and Scott E Hudson. 2009. Providing dynamically changeable physical buttons on a visual display. In Proceedings of the SIGCHI Conference on Human Factors in Computing Systems (CHI '09). ACM, 299-308.

[30] Sunao Hashimoto, Ryohei Suzuki, Youichi Kamiyama, Masahiko Inami, and Takeo Igarashi. 2013. LightCloth: senseable illuminating optical fiber cloth for creating interactive surfaces. In Proceedings of the SIGCHI Conference on Human Factors in Computing Systems (CHI '13). ACM, 603-606.

[31] Isak Herman, Leonardo Impett, Patrick KA Wollner, and Alan F Blackwell. 2015. Augmenting Bioacoustic Cognition with Tangible User Interfaces. In Foundations of Augmented Cognition. Springer, 437-448.

[32] David Holman, Jesse Burstyn, Ryan Brotman, Audrey Younkin, and Roel Vertegaal. 2013. Flexkit: a rapid prototyping platform for flexible displays. In Proceedings of the adjunct publication of the 26th annual ACM symposium on User interface software and technology (UIST '13). ACM, 17-18.

[33] David Holman and Roel Vertegaal. 2008. Organic user interfaces: designing computers in any way, shape, or form. Commun. ACM 51, 6 (2008), 48-55. 
[34] David Holman, Roel Vertegaal, Mark Altosaar, Nikolaus Troje, and Derek Johns. 2005. Paper windows: interaction techniques for digital paper. In Proceedings of the SIGCHI conference on Human factors in computing systems (CHI '05). ACM, 591-599.

[35] Hiroshi Ishii, Dávid Lakatos, Leonardo Bonanni, and Jean-Baptiste Labrune. 2012. Radical atoms: beyond tangible bits, toward transformable materials. interactions 19, 1 (2012), 38-51.

[36] Hiroshi Ishii, Carlo Ratti, Ben Piper, Yao Wang, Assaf Biderman, and Eran Ben-Joseph. 2004. Bringing clay and sand into digital designâĂŤcontinuous tangible user interfaces. BT technology journal 22, 4 (2004), 287-299.

[37] Hiroo Iwata, Hiroaki Yano, and Naoto Ono. 2005. Volflex. In SIGGRAPH '05 Emerging Technologies. ACM, 31.

[38] Yvonne Jansen and Pierre Dragicevic. 2013. An interaction model for visualizations beyond the desktop. Visualization and Computer Graphics, IEEE Transactions on 19, 12 (2013), 2396-2405.

[39] Yvonne Jansen, Thorsten Karrer, and Jan Borchers. 2011. MudPad: tactile feedback for touch surfaces. In CHI'11 Extended Abstracts on Human Factors in Computing Systems. ACM, 323-328.

[40] Cindy Hsin-Liu Kao, Ermal Dreshaj, Judith Amores, Sang-won Leigh, Xavier Benavides, Pattie Maes, Ken Perlin, and Hiroshi Ishii. 2015. clayodor: Retrieving Scents through the Manipulation of Malleable Material. In Proceedings of the Ninth International Conference on Tangible, Embedded, and Embodied Interaction (TEI '15). ACM, 697-702.

[41] Mohammadreza Khalilbeigi, Roman Lissermann, Wolfgang Kleine, and Jürgen Steimle. 2012. FoldMe: interacting with double-sided foldable displays. In Proceedings of the Sixth International Conference on Tangible, Embedded and Embodied Interaction (TEI '12). ACM, 33-40.

[42] Mohammadreza Khalilbeigi, Roman Lissermann, Max Mühlhäuser, and Jürgen Steimle. 2011. Xpaaand: interaction techniques for rollable displays. In Proceedings of the SIGCHI Conference on Human Factors in Computing Systems (CHI '11). ACM, 2729-2732.

[43] Asif Khan. 2014. Megafaces. (2014). http://www.asif-khan.com/project/sochi-winter-olympics-2014/.

[44] Johan Kildal, Susanna Paasovaara, and Viljakaisa Aaltonen. 2012. Kinetic device: designing interactions with a deformable mobile interface. In CHI'12 Extended Abstracts on Human Factors in Computing Systems. ACM, 1871-1876.

[45] Hyunjung Kim and Woohun Lee. 2011. Kinetic tiles. In Proceedings of the SIGCHI Conference on Human Factors in Computing Systems (CHI '11). ACM, 1279-1282.

[46] Konstantin Klamka and Raimund Dachselt. 2017. IllumiPaper: Illuminated Interactive Paper. In Proceedings of the 2017 CHI Conference on Human Factors in Computing Systems. ACM, 5605-5618.

[47] Sachiko Kodama. 2008. Dynamic ferrofluid sculpture: organic shape-changing art forms. Commun. ACM 51, 6 (2008), $79-81$.

[48] Jeffrey Tzu Kwan Valino Koh, Kasun Karunanayaka, and Ryohei Nakatsu. 2013. Linetic: Technical, Usability and Aesthetic Implications of a Ferrofluid-Based Organic User Interface. In Human-Computer Interaction-INTERACT 2013. Springer, 180-195.

[49] Jeffrey Tzu Kwan Valino Koh, Kasun Karunanayaka, Jose Sepulveda, Mili John Tharakan, Manoj Krishnan, and Adrian David Cheok. 2011. Liquid interface: a malleable, transient, direct-touch interface. Computers in Entertainment (CIE) 9, 2 (2011), 7.

[50] Matthijs Kwak, Kasper Hornbæk, Panos Markopoulos, and Miguel Bruns Alonso. 2014. The design space of shapechanging interfaces: a repertory grid study. In Proceedings of the 2014 conference on Designing interactive systems (DIS '14). ACM, 181-190.

[51] Ki-Uk Kyung, Jeong Mook Lim, Yo-An Lim, Suntak Park, Seung Koo Park, Inwook Hwang, Seungmoon Choi, Jongman Seo, Sang-Youn Kim, Tae-Heon Yang, et al. 2011. TAXEL: Initial progress toward self-morphing visio-haptic interface. In World Haptics Conference (WHC), 2011 IEEE. IEEE, 37-42.

[52] Byron Lahey, Audrey Girouard, Winslow Burleson, and Roel Vertegaal. 2011. PaperPhone: understanding the use of bend gestures in mobile devices with flexible electronic paper displays. In Proceedings of the SIGCHI Conference on Human Factors in Computing Systems (CHI '11). ACM, 1303-1312.

[53] Daniel Leithinger, Sean Follmer, Alex Olwal, and Hiroshi Ishii. 2014. Physical telepresence: shape capture and display for embodied, computer-mediated remote collaboration. In Proceedings of the 27th annual ACM symposium on User Interface Software and Technology (UIST '14). ACM, 461-470.

[54] Daniel Leithinger, Sean Follmer, Alex Olwal, Samuel Luescher, Akimitsu Hogge, Jinha Lee, and Hiroshi Ishii. 2013. Sublimate: state-changing virtual and physical rendering to augment interaction with shape displays. In Proceedings of the SIGCHI Conference on Human Factors in Computing Systems (CHI '13). ACM, 1441-1450.

[55] Daniel Leithinger, David Lakatos, Anthony DeVincenzi, Matthew Blackshaw, and Hiroshi Ishii. 2011. Direct and gestural interaction with relief: a $2.5 \mathrm{D}$ shape display. In Proceedings of the 24th annual ACM symposium on User interface software and technology (UIST '11). ACM, 541-548.

[56] Julian Lepinski and Roel Vertegaal. 2011. Cloth displays: interacting with drapable textile screens. In Proceedings of the fifth international conference on Tangible, embedded, and embodied interaction (TEI '11). ACM, 285-288. 
[57] David Lindlbauer, Jens Emil Grønbæk, Morten Birk, Kim Halskov, Marc Alexa, and Jörg Müller. 2016. Combining Shape-Changing Interfaces and Spatial Augmented Reality Enables Extended Object Appearance. In Proceedings of the 2016 CHI Conference on Human Factors in Computing Systems. ACM, 791-802.

[58] Julia Lo and Audrey Girouard. 2014. Fabricating Bendy: Design and Development of Deformable Prototypes. Pervasive Computing, IEEE 13, 3 (2014), 40-46.

[59] Yukiko Makino and Yasuaki Kakehi. 2011. Metamorphic light: a tabletop tangible interface using deformation of plain paper. In ACM SIGGRAPH 2011 Posters. ACM, 48.

[60] Viktor Miruchna, Robert Walter, David Lindlbauer, Maren Lehmann, Regine Von Klitzing, and Jörg Müller. 2015. GelTouch: Localized Tactile Feedback Through Thin, Programmable Gel. In Proceedings of the 28th Annual ACM Symposium on User Interface Software \& Technology. ACM, 3-10.

[61] Randall Munroe. 2012. XKCD. (2012). https://xkcd.com/924/.

[62] Ken Nakagaki, Artem Dementyev, Sean Follmer, Joseph A Paradiso, and Hiroshi Ishii. 2016. ChainFORM: A Linear Integrated Modular Hardware System for Shape Changing Interfaces. In Proceedings of the 29th Annual Symposium on User Interface Software and Technology. ACM, 87-96.

[63] Kosuke Nakajima, Yuichi Itoh, Yusuke Hayashi, Kazuaki Ikeda, Kazuyuki Fujita, and Takao Onoye. 2013. Emoballoon. In Advances in Computer Entertainment (ACE '13). Springer, 182-197.

[64] Kosuke Nakajima, Yuichi Itoh, Takayuki Tsukitani, Kazuyuki Fujita, Kazuki Takashima, Yoshifumi Kitamura, and Fumio Kishino. 2011. FuSA touch display: a furry and scalable multi-touch display. In Proceedings of the ACM International Conference on Interactive Tabletops and Surfaces (ITS '11). ACM, 35-44.

[65] Masashi Nakatani, Hiroyuki Kajimoto, Kevin Vlack, Dairoku Sekiguchi, Naoki Kawakami, and Susumu Tachi. 2005. Control method for a 3D form display with coil-type shape memory alloy. In Proceedings of the 2005 IEEE International Conference on Robotics and Automation (ICRA '05). IEEE, 1332-1337.

[66] Akira Nakayasu. 2016. Luminescent Tentacles: A Scalable SMA Motion Display. In Proceedings of the 29th Annual Symposium on User Interface Software and Technology. ACM, 33-34.

[67] Vinh P Nguyen, Sang Ho Yoon, Ansh Verma, and Karthik Ramani. 2014. Bendid: Flexible interface for localized deformation recognition. In Proceedings of the 2014 ACM International foint Conference on Pervasive and Ubiquitous Computing (UBICOMP '14). ACM, 553-557.

[68] Toshio Nojima, Yoshiharu Ooide, and Hitoshi Kawaguchi. 2013. Hairlytop interface: an interactive surface display comprised of hair-like soft actuators. In World Haptics Conference (WHC '13). IEEE, 431-435.

[69] Mie Nørgaard, Tim Merritt, Majken Kirkegaard Rasmussen, and Marianne Graves Petersen. 2013. Exploring the design space of shape-changing objects: imagined physics. In Proceedings of the 6th International Conference on Designing Pleasurable Products and Interfaces (DPPI '13). ACM, 251-260.

[70] Masa Ogata and Masaaki Fukumoto. 2015. FluxPaper: Reinventing Paper with Dynamic Actuation Powered by Magnetic Flux. In Proceedings of the 33rd Annual ACM Conference on Human Factors in Computing Systems (CHI '15). ACM, 29-38.

[71] Simon Olberding, Michael Wessely, and Jürgen Steimle. 2014. Printscreen: fabricating highly customizable thin-film touch-displays. In Proceedings of the 27th annual ACM symposium on User interface software and technology (UIST '14). ACM, 281-290.

[72] Jifei Ou, Lining Yao, Daniel Tauber, Jürgen Steimle, Ryuma Niiyama, and Hiroshi Ishii. 2014. jamSheets: thin interfaces with tunable stiffness enabled by layer jamming. In Proceedings of the 8th International Conference on Tangible, Embedded and Embodied Interaction (TEI '14). ACM, 65-72.

[73] Joshua Peschke, Fabian Göbel, Thomas Gründer, Mandy Keck, Dietrich Kammer, and Rainer Groh. 2012. DepthTouch: an elastic surface for tangible computing. In Proceedings of the International Working Conference on Advanced Visual Interfaces (AVI '12). ACM, 770-771.

[74] Ben Piper, Carlo Ratti, and Hiroshi Ishii. 2002. Illuminating clay: a 3-D tangible interface for landscape analysis. In Proceedings of the SIGCHI conference on Human factors in computing systems (CHI '02). ACM, 355-362.

[75] Ivan Poupyrev, Tatsushi Nashida, Shigeaki Maruyama, Jun Rekimoto, and Yasufumi Yamaji. 2004. Lumen: interactive visual and shape display for calm computing. In ACM SIGGRAPH 2004 Emerging technologies. ACM, 17.

[76] Ivan Poupyrev, Tatsushi Nashida, and Makoto Okabe. 2007. Actuation and tangible user interfaces: the Vaucanson duck, robots, and shape displays. In Proceedings of the 1st international conference on Tangible and embedded interaction (TEI '07). ACM, 205-212.

[77] Raf Ramakers, Johannes Schöning, and Kris Luyten. 2014. Paddle: highly deformable mobile devices with physical controls. In Proceedings of the 32nd annual ACM conference on Human factors in computing systems (CHI '14). ACM, 2569-2578.

[78] Majken Kirkegaard Rasmussen, Erik Grönvall, Sofie Kinch, and Marianne Graves Petersen. 2013. It's alive, it's magic, it's in love with you: opportunities, challenges and open questions for actuated interfaces. In Proceedings of the 25th Australian Computer-Human Interaction Conference: Augmentation, Application, Innovation, Collaboration. ACM, 
63-72.

[79] Majken K Rasmussen, Esben W Pedersen, Marianne G Petersen, and Kasper Hornbæk. 2012. Shape-changing interfaces: a review of the design space and open research questions. In Proceedings of the SIGCHI Conference on Human Factors in Computing Systems (CHI '12). ACM, 735-744.

[80] Anne Roudaut, Abhijit Karnik, Markus Löchtefeld, and Sriram Subramanian. 2013. Morphees: toward high shape resolution in self-actuated flexible mobile devices. In Proceedings of the SIGCHI Conference on Human Factors in Computing Systems (CHI '13). ACM, 593-602.

[81] Anne Roudaut, Rebecca Reed, Tianbo Hao, and Sriram Subramanian. 2014. Changibles: analyzing and designing shape changing constructive assembly. In Proceedings of the 32nd annual ACM conference on Human factors in computing systems (CHI '14). ACM, 2593-2596.

[82] Aimee Rydarowski, Ozge Samanci, and Ali Mazalek. 2008. Murmur: kinetic relief sculpture, multi-sensory display, listening machine. In Proceedings of the 2nd international conference on Tangible and embedded interaction (TEI' 08). ACM, 231-238.

[83] Deepak Sahoo, Kasper Hornbæk, and Sriram Subramanian. 2016. TableHop: an actuated fabric display using transparent electrodes. (2016), 00-00.

[84] Takao Sato, Jefferson Pardomuan, Yasushi Matoba, and Hideaki Koike. 2014. ClaytricSurface: An Interactive Deformable Display with Dynamic Stiffness Control. Computer Graphics and Applications, IEEE 34, 3 (2014), 59-67.

[85] Magdalena Schmid, Sonja Rümelin, and Hendrik Richter. 2013. Empowering materiality: inspiring the design of tangible interactions. In Proceedings of the 7th International Conference on Tangible, Embedded and Embodied Interaction (TEI '13). ACM, 91-98.

[86] Holger Schnädelbach, Kevin Glover, and Ainojie Alexander Irune. 2010. ExoBuilding: breathing life into architecture. In Proceedings of the 6th Nordic Conference on Human-Computer Interaction: Extending Boundaries (NordiCHI '10). ACM, 442-451.

[87] Sue Ann Seah, Marianna Obrist, Anne Roudaut, and Sriram Subramanian. 2015. Need for Touch in Human Space Exploration: Towards the Design of a Morphing Haptic Glove-ExoSkin. In Human-Computer Interaction-INTERACT 2015. Springer, 18-36.

[88] Brian K Smith. 2006. Design and computational flexibility. Digital Creativity 17, 2 (2006), 65-72.

[89] Jürgen Steimle, Andreas Jordt, and Pattie Maes. 2013. Flexpad: highly flexible bending interactions for projected handheld displays. In Proceedings of the SIGCHI Conference on Human Factors in Computing Systems (CHI '13). ACM, 237-246.

[90] Andrew Stevenson, Christopher Perez, and Roel Vertegaal. 2011. An inflatable hemispherical multi-touch display. In Proceedings of the fifth international conference on Tangible, embedded, and embodied interaction (TEI '11). ACM, 289-292.

[91] Paul Strohmeier, Jesse Burstyn, Juan Pablo Carrascal, Vincent Levesque, and Roel Vertegaal. 2016. ReFlex: A Flexible Smartphone with Active Haptic Feedback for Bend Input. In Proceedings of the TEI'16: Tenth International Conference on Tangible, Embedded, and Embodied Interaction. ACM, 185-192.

[92] Miriam Sturdee, John Hardy, Nick Dunn, and Jason Alexander. 2015. A Public Ideation of Shape-Changing Applications. In Proceedings of the 2015 International Conference on Interactive Tabletops \& Surfaces. ACM, 219-228.

[93] Faisal Taher, John Hardy, Abhijit Karnik, Christian Weichel, Yvonne Jansen, Kasper Hornbæk, and Jason Alexander. 2015. Exploring interactions with physically dynamic bar charts. In Proceedings of the 33rd Annual ACM Conference on Human Factors in Computing Systems (CHI '15). ACM, 3237-3246.

[94] Faisal Taher, John Vidler, and Jason Alexander. 2016. A Characterization of Actuation Techniques for Generating Movement in Shape-Changing Interfaces. In International fournal of Human-Computer Interaction. ACM, XX-XX.

[95] Kazuki Takashima, Naohiro Aida, Hitomi Yokoyama, and Yoshifumi Kitamura. 2013. TransformTable: a self-actuated shape-changing digital table. In Proceedings of the 2013 ACM international conference on Interactive tabletops and surfaces (ITS '13). ACM, 179-188.

[96] Kazuki Takashima, Takafumi Oyama, Yusuke Asari, Ehud Sharlin, Saul Greenberg, and Yoshifumi Kitamura. 2016. Study and Design of a Shape-Shifting Wall Display. In Proceedings of the 2016 ACM Conference on Designing Interactive Systems. ACM, 796-806.

[97] Dominique Tan, Maciej Kumorek, Andres A Garcia, Adam Mooney, and Derek Bekoe. 2015. Projectagami: A foldable mobile device with shape interactive applications. In Proceedings of the 33rd Annual ACM Conference Extended Abstracts on Human Factors in Computing Systems. ACM, 1555-1560.

[98] Aneesh P Tarun, Byron Lahey, Audrey Girouard, Winslow Burleson, and Roel Vertegaal. 2011. Snaplet: using body shape to inform function in mobile flexible display devices. In CHI'11 Extended Abstracts on Human Factors in Computing Systems. ACM, 329-334.

[99] Aneesh P Tarun, Peng Wang, Audrey Girouard, Paul Strohmeier, Derek Reilly, and Roel Vertegaal. 2013. PaperTab: an electronic paper computer with multiple large flexible electrophoretic displays. In CHI'13 Extended Abstracts on 
Human Factors in Computing Systems. ACM, 3131-3134.

[100] Giovanni Maria Troiano, Esben Warming Pedersen, and Kasper Hornbæk. 2014. User-defined gestures for elastic, deformable displays. In Proceedings of the 2014 International Working Conference on Advanced Visual Interfaces (AVI '14). ACM, 1-8.

[101] Jessica Tsimeris, Colin Dedman, Michael Broughton, and Tom Gedeon. 2013. ForceForm: a dynamically deformable interactive surface. In Proceedings of the 2013 ACM international conference on Interactive tabletops and surfaces (ITS '13). ACM, 175-178.

[102] Anna Vallgårda. 2014. Giving form to computational things: developing a practice of interaction design. Personal and ubiquitous computing 18, 3 (2014), 577-592.

[103] Florian Vogt, Timothy Chen, Reynald Hoskinson, and Sidney Fels. 2004. A malleable surface touch interface. In ACM SIGGRAPH 2004 Sketches. ACM, 36.

[104] Akira Wakita and Akito Nakano. 2012. Blob manipulation. In Proceedings of the Sixth International Conference on Tangible, Embedded and Embodied Interaction (TEI '12). ACM, 299-302.

[105] Jun-ichiro Watanabe, Arito Mochizuki, and Youichi Horry. 2008. Bookisheet: bendable device for browsing content using the metaphor of leafing through the pages. In Proceedings of the 10th international conference on Ubiquitous computing (Ubicomp '08). ACM, 360-369.

[106] Yoshihiro Watanabe, Alvaro Cassinelli, Takashi Komuro, and Masatoshi Ishikawa. 2008. The deformable workspace: A membrane between real and virtual space. In 3rd IEEE International Workshop onHorizontal Interactive Human Computer Systems (TABLETOP '08). IEEE, 145-152.

[107] Christian Weichel, Jason Alexander, and John Hardy. 2015. Shape Display Shader Language (SDSL): A New Programming Model for Shape Changing Displays. In Proceedings of the 33rd Annual ACM Conference Extended Abstracts on Human Factors in Computing Systems (CHI '15 EA). ACM, 1121-1126.

[108] Lining Yao, Jifei Ou, Daniel Tauber, and Hiroshi Ishii. 2014. Integrating optical waveguides for display and sensing on pneumatic soft shape changing interfaces. In Proceedings of the adjunct publication of the 27th annual ACM symposium on User interface software and technology (UIST '14). ACM, 117-118.

[109] Zi Ye and Hammad Khalid. 2010. Cobra: flexible displays for mobilegaming scenarios. In CHI'10 Extended Abstracts on Human Factors in Computing Systems. ACM, 4363-4368.

[110] Tomoyuki Yokota, Peter Zalar, Martin Kaltenbrunner, Hiroaki Jinno, Naoji Matsuhisa, Hiroki Kitanosako, Yutaro Tachibana, Wakako Yukita, Mari Koizumi, and Takao Someya. 2016. Ultraflexible organic photonic skin. Science Advances 2, 4 (2016).

[111] Kyungwon Yun, JunBong Song, Keehong Youn, Sungmin Cho, and Hyunwoo Bang. 2013. ElaScreen: exploring multi-dimensional data using elastic screen. In CHI'13 Extended Abstracts on Human Factors in Computing Systems. ACM, 1311-1316.

Received October 2016; revised September 2017; accepted September 2017 